\title{
Thin accretion disks onto brane world black holes
}

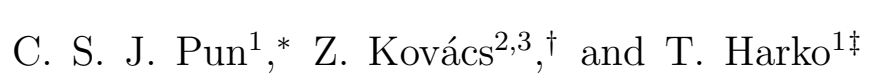 \\ ${ }^{1}$ Department of Physics and Center for Theoretical and Computational Physics, \\ The University of Hong Kong, Pok Fu Lam Road, Hong Kong \\ ${ }^{2}$ Max-Planck-Institut für Radioastronomie, \\ Auf dem Hügel 69, 53121 Bonn, Germany and \\ ${ }^{3}$ Department of Experimental Physics, \\ University of Szeged, Dóm Tér 9, Szeged 6720, Hungary
}

(Dated: October 30, 2018)

\begin{abstract}
The braneworld description of our universe entails a large extra dimension and a fundamental scale of gravity that might be lower by several orders of magnitude as compared to the Planck scale. An interesting consequence of the braneworld scenario is in the nature of the vacuum solutions of the brane gravitational field equations, with properties quite distinct as compared to the standard black hole solutions of general relativity. One possibility of observationally discriminating between different types of black holes is the study of the emission properties of the accretion disks. In the present paper we obtain the energy flux, the emission spectrum and accretion efficiency from the accretion disks around several classes of static and rotating brane world black holes, and we compare them to the general relativistic case. Particular signatures can appear in the electromagnetic spectrum, thus leading to the possibility of directly testing extra-dimensional physical models by using astrophysical observations of the emission spectra from accretion disks.
\end{abstract}

PACS numbers: 04.50.+h, 04.20.Jb, 04.20.Cv, 95.35.+d

\footnotetext{
*Electronic address: jcspun@hkucc.hku.hk

${ }^{\dagger}$ Electronic address: zkovacs@mpifr-bonn.mpg.de

‡Electronic address: harko@hkucc.hku.hk
} 


\section{INTRODUCTION}

The idea, proposed in [1, 2], that our four-dimensional Universe might be a three-brane, embedded in a five-dimensional space-time (the bulk), has attracted a considerable interest in the past few years. According to the brane-world scenario, the physical fields (electromagnetic, Yang-Mills etc.) in our four-dimensional Universe are confined to the three brane. These fields are assumed to arise as fluctuations of branes in string theories. Only gravity can freely propagate in both the brane and bulk space-times, with the gravitational self-couplings not significantly modified. This model originated from the study of a single 3-brane embedded in five dimensions, with the $5 D$ metric given by $d s^{2}=e^{-f(y)} \eta_{\mu \nu} d x^{\mu} d x^{\nu}+d y^{2}$, which, due to the appearance of the warp factor, could produce a large hierarchy between the scale of particle physics and gravity. Even if the fifth dimension is uncompactified, standard $4 D$ gravity is reproduced on the brane. Hence this model allows the presence of large, or even infinite non-compact extra dimensions. Our brane is identified to a domain wall in a 5-dimensional anti-de Sitter space-time. For a review of the brane world models see [3].

The braneworld description of our universe entails a large extra dimension and a fundamental scale of gravity that might be lower by several orders of magnitude compared to the Planck scale [1, 2]. Due to the correction terms coming from the extra dimensions, significant deviations from the Einstein theory occur in brane world models at very high energies [4, 5]. Gravity is largely modified at the electro-weak scale of $1 \mathrm{TeV}$. The cosmological and astrophysical implications of the brane world theories have been extensively investigated in the physical literature [6, 7].

Several classes of spherically symmetric solutions of the static gravitational field equations in the vacuum on the brane have been obtained in [8, 9, 10, 11]. As a possible physical application of these solutions the behavior of the angular velocity $v_{t g}$ of the test particles in stable circular orbits has been considered [9, 10, 11]. The general form of the solution, together with two constants of integration, uniquely determines the rotational velocity of the particle. In the limit of large radial distances, and for a particular set of values of the integration constants the angular velocity tends to a constant value. This behavior is typical for massive particles (hydrogen clouds) outside galaxies, and is usually explained by postulating the existence of the dark matter. The exact galactic metric, the dark radiation, the dark pressure and the lensing in the flat rotation curves region in the brane world scenario 
has been obtained in [11].

For standard general relativistic spherical compact objects the exterior space-time is described by the Schwarzschild metric. In the five dimensional brane world models, the high energy corrections to the energy density, together with the Weyl stresses from bulk gravitons, imply that on the brane the exterior metric of a static star is no longer the Schwarzschild metric [12]. The presence of the Weyl stresses also means that the matching conditions do not have a unique solution on the brane; the knowledge of the five-dimensional Weyl tensor is needed as a minimum condition for uniqueness.

It is known that the Einstein field equations in five dimensions admit more general spherically symmetric black holes on the brane than four-dimensional general relativity. Hence an interesting consequence of the braneworld scenario is in the nature of the spherically symmetric vacuum solutions to the brane gravitational field equations, which could represent black holes with properties quite distinct as compared to ordinary black holes in four dimensions. Such black holes are likely to have very diverse cosmological and astrophysical signatures. Static, spherically symmetric exterior vacuum solutions of the brane world models have been proposed first in [12] and in [13]. The first of these solutions, obtained in [12], has the mathematical form of the Reissner-Nordström solution of the standard general relativity, in which a tidal Weyl parameter plays the role of the electric charge of the general relativistic solution. The solution has been obtained by imposing the null energy condition on the 3-brane for a bulk having non zero Weyl curvature, and it can be matched to the interior solution corresponding to a constant density brane world star. A second exterior solution, which also matches a constant density interior, has been derived in [13].

Two families of analytic solutions of the spherically symmetric vacuum brane world model equations (with $g_{t t} \neq-1 / g_{r r}$ ), parameterized by the ADM mass and a PPN parameter $\beta$ have been obtained in [14]. Non-singular black-hole solutions in the brane world model have been considered in [15], by relaxing the condition of the zero scalar curvature but retaining the null energy condition. The four-dimensional Gauss and Codazzi equations for an arbitrary static spherically symmetric star in a Randall-Sundrum type II brane world have been completely solved on the brane in [16]. The on-brane boundary can be used to determine the full 5-dimensional space-time geometry. The procedure can be generalized to solid objects such as planets.

A method to extend into the bulk asymptotically flat static spherically symmetric brane- 
world metrics has been proposed in [17]. The exact integration of the field equations along the fifth coordinate was done by using the multipole $(1 / r)$ expansion. The results show that the shape of the horizon of the brane black hole solutions is very likely a flat "pancake" for astrophysical sources.

The general solution to the trace of the 4-dimensional Einstein equations for static, spherically symmetric configurations has been used as a basis for finding a general class of black hole metrics, containing one arbitrary function $g_{t t}=A(r)$, which vanishes at some $r=r_{h}>0$ (the horizon radius) in [18]. Under certain reasonable restrictions, black hole metrics are found, with or without matter. Depending on the boundary conditions the metrics can be asymptotically flat, or have any other prescribed asymptotic. The exact stationary and axisymmetric solutions describing charged rotating black holes localized on a 3-brane in the Randall-Sundrum braneworld were studied in [19]. By taking the metric on the brane to be of the Kerr-Schild form it can be shown that the Kerr-Newman solution of ordinary general relativity in which the electric charge is superseded by a tidal charge satisfies a closed system of the effective gravitational field equations on the brane. The negative tidal charge may provide a mechanism for spinning up the black hole so that its rotation parameter exceeds its mass. For a review of the black hole properties and of the lensing in the brane world models see [20].

It is generally expected that most of the astrophysical objects grow substantially in mass via accretion. Recent observations suggest that around most of the active galactic nuclei (AGN's) or black hole candidates there exist gas clouds surrounding the central compact object, and an associated accretion disc, on a variety of scales from a tenth of a parsec to a few hundred parsecs [21]. These clouds are assumed to form a geometrically and optically thick torus (or warped disc), which absorbs most of the ultraviolet radiation and the soft X-rays. The gas exists in either the molecular or the atomic phase. The most powerful evidence for the existence of super massive black holes comes from the very-long baseline interferometry (VLBI) imaging of molecular $\mathrm{H}_{2} \mathrm{O}$ masers in the active galaxy NGC 4258 [22]. This imaging, produced by Doppler shift measurements assuming Keplerian motion of the masering source, has allowed a quite accurate estimation of the central mass, which has been found to be a $3.6 \times 10^{7} M_{\odot}$ super massive dark object, within 0.13 parsecs. Hence, important astrophysical information can be obtained from the observation of the motion of the gas streams in the gravitational field of compact objects. 
The determination of the accretion rate for an astrophysical object can give a strong evidence for the existence of a surface of the object. A model in which Sgr A*, the $3.7 \times$ $10^{6} M_{\odot}$ super massive black hole candidate at the Galactic center, may be a compact object with a thermally emitting surface was considered in [23]. For very compact surfaces within the photon orbit, the thermal assumption is likely to be a good approximation because of the large number of rays that are strongly gravitationally lensed back onto the surface. Given the very low quiescent luminosity of Sgr A* in the near-infrared, the existence of a hard surface, even in the limit in which the radius approaches the horizon, places a severe constraint on the steady mass accretion rate onto the source, $\dot{M} \leq 10^{-12} M_{\odot} \mathrm{yr}^{-1}$. This limit is well below the minimum accretion rate needed to power the observed submillimeter luminosity of $\operatorname{Sgr} \mathrm{A}^{*}, \dot{M} \geq 10^{-10} M_{\odot} \mathrm{yr}$.

Thus, from the determination of the accretion rate it follows that Sgr A* does not have a surface, that is, it must have an event horizon. Therefore the study of the accretion processes by compact objects is a powerful indicator of their physical nature.

The first comprehensive theory of accretion disks around black holes was constructed in [24]. This theory was extended to the general relativistic models of the mass accretion onto rotating black holes in [25]. These pioneering works developed thin steady-state accretion disks, where the accreting matter moves in Keplerian orbits. The hydrodynamical equilibrium in the disk is maintained by an efficient cooling mechanism via radiation transport. The photon flux emitted by the disk surface was studied under the assumption that the disk emits a black body radiation. The properties of radiant energy flux over the thin accretion disks were further analyzed in [26] and in [27], where the effects of the photon capture by the hole on the spin evolution were presented as well. In these works the efficiency with which black holes convert rest mass into outgoing radiation in the accretion process was also computed.

The emissivity properties of the accretion disks have also been investigated for exotic central objects recently, such as quark, boson or fermion stars for both rotating and nonrotating cases [28, 29, 30], as well as for the modified $f(R)$ type theories of gravity [31]. The radiation power per unit area, the temperature of the disk and the spectrum of the emitted radiation were given, and compared with the case of a Schwarzschild black hole of an equal mass.

It is the purpose of the present paper to study the matter accretion by brane world black 
holes. By using the general formalism of accretion we analyze the accretion process for several black hole type solutions of the gravitational field equations on the brane, both nonrotating and rotating, which have been previously obtained. Particular signatures can appear in the electromagnetic spectrum, thus leading to the possibility of directly testing extradimensional physical models by using astrophysical observations of the emission spectra from accretion disks.

The present paper is organized as follows. We review the field equations of the brane world models and the static, spherically symmetric solutions of the field equations as well as the rotating ones in Section II. The thin accretion disks onto black holes are briefly described in Section III. In Section IV we consider the radiation flux, spectrum and efficiency of thin accretion disks onto several classes of brane world black holes. We discuss and conclude our results in Section V.

\section{THE GRAVITATIONAL FIELD EQUATIONS IN THE BRANE WORLD MODELS}

In the present Section we briefly describe the basic mathematical formalism of the brane world models, and we present the spherically symmetric static vacuum field equations. The solutions of the vacuum field equations on the brane physically describe the brane world black holes.

\section{A. The gravitational field equations on the brane}

We start by considering a five dimensional (5D) spacetime (the bulk), with a single fourdimensional (4D) brane, on which matter is confined. The $4 \mathrm{D}$ brane world $\left({ }^{(4)} M, g_{\mu \nu}\right)$ is located at a hypersurface $\left(B\left(X^{A}\right)=0\right)$ in the $5 \mathrm{D}$ bulk spacetime $\left({ }^{(5)} M, g_{A B}\right)$, of which coordinates are described by $X^{A}, A=0,1, \ldots, 4$. The induced $4 \mathrm{D}$ coordinates on the brane are $x^{\mu}, \mu=0,1,2,3$. In the present paper the capital Latin indices $A, B, \ldots, I, J, \ldots$ take values in the range $0,1, \ldots, 4$, while the Greek indices run in the range $0, \ldots, 3$.

The action of the system is given by $S=S_{\text {bulk }}+S_{\text {brane }}$, where

$$
S_{b u l k}=\int_{(5) M} \sqrt{-{ }^{(5)} g}\left[\frac{1}{2 k_{5}^{2}}{ }^{(5)} R+{ }^{(5)} L_{m}+\Lambda_{5}\right] d^{5} X
$$


and

$$
S_{\text {brane }}=\int_{(4) M} \sqrt{-^{(5)} g}\left[\frac{1}{k_{5}^{2}} K^{ \pm}+L_{\text {brane }}\left(g_{\alpha \beta}, \psi\right)+\lambda_{b}\right] d^{4} x,
$$

where $k_{5}^{2}=8 \pi G_{5}$ is the $5 \mathrm{D}$ gravitational constant, ${ }^{(5)} R$ and ${ }^{(5)} L_{m}$ are the $5 \mathrm{D}$ scalar curvature and the matter Lagrangian in the bulk, $L_{b r a n e}\left(g_{\alpha \beta}, \psi\right)$ is the $4 \mathrm{D}$ Lagrangian, which is given by a generic functional of the brane metric $g_{\alpha \beta}$ and of the matter fields $\psi, K^{ \pm}$is the trace of the extrinsic curvature on either side of the brane, and $\Lambda_{5}$ and $\lambda_{b}$ (the constant brane tension) are the negative vacuum energy densities in the bulk and on the brane, respectively [4].

The Einstein field equations in the bulk are given by [4]

$$
{ }^{(5)} G_{I J}=k_{5}^{2(5)} T_{I J}, \quad{ }^{(5)} T_{I J}=-\Lambda_{5}{ }^{(5)} g_{I J}+\delta(B)\left[-\lambda_{b}{ }^{(5)} g_{I J}+T_{I J}\right]
$$

where ${ }^{(5)} T_{I J} \equiv-2 \delta^{(5)} L_{m} / \delta^{(5)} g^{I J}+{ }^{(5)} g_{I J}(5) L_{m}$, is the energy-momentum tensor of bulk matter fields, while $T_{\mu \nu}$ is the energy-momentum tensor localized on the brane and which is defined by $T_{\mu \nu} \equiv-2 \delta L_{\text {brane }} / \delta g^{\mu \nu}+g_{\mu \nu} L_{\text {brane }}$.

The delta function $\delta(B)$ denotes the localization of brane contribution. In the 5D spacetime a brane is a fixed point of the $Z_{2}$ symmetry. The basic equations on the brane are obtained by projections onto the brane world. The induced $4 \mathrm{D}$ metric is $g_{I J}={ }^{(5)} g_{I J}-n_{I} n_{J}$, where $n_{I}$ is the space-like unit vector field normal to the brane hypersurface ${ }^{(4)} M$. The unit vector field satisfies the condition $n_{I} n^{I}=-1$. In the following we assume ${ }^{(5)} L_{m}=0$. In the brane world models only gravity can probe the extra dimensions.

Assuming a metric of the form $d s^{2}=\left(n_{I} n_{J}+g_{I J}\right) d x^{I} d x^{J}$, with $n_{I} d x^{I}=d \chi$ the unit normal to the $\chi=$ constant hypersurfaces and $g_{I J}$ the induced metric on $\chi=$ constant hypersurfaces, the effective $4 \mathrm{D}$ gravitational equation on the brane takes the form [4]:

$$
G_{\mu \nu}=-\Lambda g_{\mu \nu}+k_{4}^{2} T_{\mu \nu}+k_{5}^{4} S_{\mu \nu}-E_{\mu \nu}
$$

where $S_{\mu \nu}$ is the local quadratic energy-momentum correction

$$
S_{\mu \nu}=\frac{1}{12} T T_{\mu \nu}-\frac{1}{4} T_{\mu}^{\alpha} T_{\nu \alpha}+\frac{1}{24} g_{\mu \nu}\left(3 T^{\alpha \beta} T_{\alpha \beta}-T^{2}\right)
$$

and $E_{\mu \nu}$ is the non-local effect from the free bulk gravitational field, the transmitted projection of the bulk Weyl tensor $C_{I A J B}, E_{I J}=C_{I A J B} n^{A} n^{B}$, with the property $E_{I J} \rightarrow$ $E_{\mu \nu} \delta_{I}^{\mu} \delta_{J}^{\nu} \quad$ as $\quad \chi \rightarrow 0$. We have also denoted $k_{4}^{2}=8 \pi G$, with $G$ the usual $4 \mathrm{D}$ gravitational constant. 
The $4 \mathrm{D}$ cosmological constant, $\Lambda$, and the $4 \mathrm{D}$ coupling constant, $k_{4}$, are related by $\Lambda=k_{5}^{2}\left(\Lambda_{5}+k_{5}^{2} \lambda_{b}^{2} / 6\right) / 2$ and $k_{4}^{2}=k_{5}^{4} \lambda_{b} / 6$, respectively. In the limit $\lambda_{b}^{-1} \rightarrow 0$ we recover standard general relativity [4].

The Einstein equation in the bulk and the Codazzi equation also imply the conservation of the energy-momentum tensor of the matter on the brane, $D_{\nu} T_{\mu}{ }^{\nu}=0$, where $D_{\nu}$ denotes the brane covariant derivative. Moreover, from the contracted Bianchi identities on the brane it follows that the projected Weyl tensor obeys the constraint $D_{\nu} E_{\mu}{ }^{\nu}=k_{5}^{4} D_{\nu} S_{\mu}{ }^{\nu}$.

The symmetry properties of $E_{\mu \nu}$ imply that in general we can decompose it irreducibly with respect to a chosen 4 -velocity field $u^{\mu}$ as $E_{\mu \nu}=$ $-k^{4}\left[U\left(u_{\mu} u_{\nu}+\frac{1}{3} h_{\mu \nu}\right)+P_{\mu \nu}+2 Q_{(\mu} u_{\nu)}\right]$, where $k=k_{5} / k_{4}, h_{\mu \nu}=g_{\mu \nu}+u_{\mu} u_{\nu}$ projects orthogonal to $u^{\mu}$, the "dark radiation" term $U=-k^{-4} E_{\mu \nu} u^{\mu} u^{\nu}$ is a scalar, $Q_{\mu}=k^{-4} h_{\mu}^{\alpha} E_{\alpha \beta} u^{\beta}$ is a spatial vector and $P_{\mu \nu}=-k^{-4}\left[h_{(\mu}{ }^{\alpha} h_{\nu)}{ }^{\beta}-\frac{1}{3} h_{\mu \nu} h^{\alpha \beta}\right] E_{\alpha \beta}$ is a spatial, symmetric and trace-free tensor [3].

In the case of the vacuum state we have $\rho=p=0, T_{\mu \nu} \equiv 0$, and consequently $S_{\mu \nu} \equiv 0$. Therefore the field equation describing a static brane takes the form

$$
R_{\mu \nu}=-E_{\mu \nu}+\Lambda g_{\mu \nu}
$$

with the trace $R$ of the Ricci tensor $R_{\mu \nu}$ satisfying the condition $R=R_{\mu}^{\mu}=4 \Lambda$.

In the vacuum case $E_{\mu \nu}$ satisfies the constraint $D_{\nu} E_{\mu}{ }^{\nu}=0$. In an inertial frame at any point on the brane we have $u^{\mu}=\delta_{0}^{\mu}$ and $h_{\mu \nu}=\operatorname{diag}(0,1,1,1)$. In a static vacuum $Q_{\mu}=0$ and the constraint for $E_{\mu \nu}$ takes the form [13]

$$
\frac{1}{3} D_{\mu} U+\frac{4}{3} U A_{\mu}+D^{\nu} P_{\mu \nu}+A^{\nu} P_{\mu \nu}=0
$$

where $A_{\mu}=u^{\nu} D_{\nu} u_{\mu}$ is the 4 -acceleration. In the static spherically symmetric case we may chose $A_{\mu}=A(r) r_{\mu}$ and $P_{\mu \nu}=P(r)\left(r_{\mu} r_{\nu}-\frac{1}{3} h_{\mu \nu}\right)$, where $A(r)$ and $P(r)$ (the "dark pressure") are some scalar functions of the radial distance $r$, and $r_{\mu}$ is a unit radial vector [12].

\section{B. Static and spherically symmetric brane world black holes}

Static black holes are described by the static and spherically symmetric metric given by

$$
d s^{2}=-e^{\nu(r)} d t^{2}+e^{\lambda(r)} d r^{2}+r^{2}\left(d \theta^{2}+\sin ^{2} \theta d \phi^{2}\right) .
$$


With the metric given by (7) the gravitational field equations and the effective energymomentum tensor conservation equation in the vacuum take the form [8, 9]

$$
\begin{gathered}
-e^{-\lambda}\left(\frac{1}{r^{2}}-\frac{\lambda^{\prime}}{r}\right)+\frac{1}{r^{2}}=3 \alpha U+\Lambda, \\
e^{-\lambda}\left(\frac{\nu^{\prime}}{r}+\frac{1}{r^{2}}\right)-\frac{1}{r^{2}}=\alpha(U+2 P)-\Lambda, \\
\frac{1}{2} e^{-\lambda}\left(\nu^{\prime \prime}+\frac{\nu^{\prime 2}}{2}+\frac{\nu^{\prime}-\lambda^{\prime}}{r}-\frac{\nu^{\prime} \lambda^{\prime}}{2}\right)=\alpha(U-P)-\Lambda, \\
\nu^{\prime}=-\frac{U^{\prime}+2 P^{\prime}}{2 U+P}-\frac{6 P}{r(2 U+P)},
\end{gathered}
$$

where $^{\prime}=d / d r$, and we have denoted $\alpha=16 \pi G / k^{4} \lambda_{b}$.

The field equations (8) -(10) can be interpreted as describing an anisotropic "matter distribution", with the effective energy density $\rho^{\text {eff }}$, radial pressure $P^{\text {eff }}$ and orthogonal pressure $P_{\perp}^{\mathrm{eff}}$, respectively, so that $\rho^{\mathrm{eff}}=3 \alpha U+\Lambda, P^{\mathrm{eff}}=\alpha U+2 \alpha P-\Lambda$ and $P_{\perp}^{\mathrm{eff}}=$ $\alpha U-\alpha P-\Lambda$, respectively, which gives the condition $\rho^{\mathrm{eff}}-P^{\mathrm{eff}}-2 P_{\perp}^{\mathrm{eff}}=4 \Lambda=$ constant. This is expected for the 'radiation' like source, for which the projection of the bulk Weyl tensor is trace-less, $E_{\mu}^{\mu}=0$.

Eq. (8) can immediately be integrated to give

$$
e^{-\lambda}=1-\frac{C_{1}}{r}-\frac{G M_{U}(r)}{r}-\frac{\Lambda}{3} r^{2},
$$

where $C_{1}$ is an arbitrary constant of integration, and we denoted $G M_{U}(r)=3 \alpha \int_{0}^{r} U(r) r^{2} d r$.

The function $M_{U}$ is the gravitational mass corresponding to the dark radiation term (the dark mass). For $U=0$ the metric coefficient given by Eq. (12) must tend to the standard general relativistic Schwarzschild metric coefficient, which gives $C_{1}=2 G M$, where $M=$ constant is the baryonic (usual) mass of the gravitating system.

By substituting $\nu^{\prime}$ given by Eq. (11) into Eq. (9), and with the use of Eq. (12), we obtain the following system of differential equations satisfied by the dark radiation term $U$, the dark pressure $P$ and the dark mass $M_{U}$, describing the vacuum gravitational field, exterior to a massive body, in the brane world model [8]]:

$$
\begin{gathered}
\frac{d M_{U}}{d r}=\frac{3 \alpha}{G} r^{2} U \\
\frac{d U}{d r}=-\frac{(2 U+P)\left[2 G M+G M_{U}-\frac{2}{3} \Lambda r^{3}+\alpha(U+2 P) r^{3}\right]}{r^{2}\left(1-\frac{2 G M}{r}-\frac{M_{U}}{r}-\frac{\Lambda}{3} r^{2}\right)}-2 \frac{d P}{d r}-\frac{6 P}{r}
\end{gathered}
$$


To close the system a supplementary functional relation between one of the unknowns $U$, $P$ and $M_{U}$ is needed. Generally, this equation of state is given in the form $P=P(U)$. Once this relation is known, Eqs. (13)-(14) give a full description of the geometrical properties of the vacuum on the brane.

In the following we will restrict our analysis to the case $\Lambda=0$. Then the system of equations (13) and (14) can be transformed to an autonomous system of differential equations by means of the transformations $q=2 G M / r+G M_{U} / r, \mu=3 \alpha r^{2} U, p=3 \alpha r^{2} P, \theta=\ln r$ where $\mu$ and $p$ are the "reduced" dark radiation and pressure, respectively. With the use of the new variables, Eqs. (13) and (14) become

$$
\begin{gathered}
\frac{d q}{d \theta}=\mu-q, \\
\frac{d \mu}{d \theta}=-\frac{(2 \mu+p)\left[q+\frac{1}{3}(\mu+2 p)\right]}{1-q}-2 \frac{d p}{d \theta}+2 \mu-2 p .
\end{gathered}
$$

Eqs. (13) and (14), or, equivalently, (15) and (16), are called the structure equations of the vacuum on the brane [8]. In order to close the system of equations (15) and (16) an "equation of state" $p=p(\mu)$, relating the reduced dark radiation and the dark pressure terms, is needed. Once the equation of state is known, exact vacuum solutions of the gravitational field equations on the brane can be obtained. The opposite procedure can also be followed, that is, by specifying the functional form of the metric tensor, the dark radiation and the dark pressure can be obtained from the field equations. Therefore, several exact solutions of the gravitational field equations on the brane can be obtained [12, 14, 18].

\section{Rotating brane world black holes}

In order to study rotating black hole solutions on the brane it is convenient to assume that the axisymmetric and stationary metric is of the Kerr-Schild form, and can be expressed in the form of its linear approximation around the flat metric, $d s^{2}=\left(d s^{2}\right)_{\text {flat }}+H\left(l_{\mu} d x^{\mu}\right)^{2}$, where $l_{\mu}$ is a null, geodesic vector field in both the flat and full metrics, and $H$ is an arbitrary scalar function. By introducing a set of coordinates $y^{\mu}=\{u, r, \theta, \phi\}$, the metric can be written in the alternative form [19]

$$
d s^{2}=\left[-(d u+d r)^{2}+d r^{2}+\Sigma d \theta^{2}+\left(r^{2}+a^{2}\right) \sin ^{2} \theta d \phi^{2}+2 a \sin ^{2} \theta d r d \phi+H\left(d u-a \sin ^{2} \theta d \phi\right)^{2}\right]
$$


where $H=H(r, \theta)$, the parameter $a$ is related to the angular momentum of the black hole, and the quantity $\Sigma$ is defined as $\Sigma=r^{2}+a^{2} \cos ^{2} \theta$. The condition $R=0$ gives for $H$ the differential equation

$$
\left(\frac{\partial^{2}}{\partial r^{2}}+\frac{4 r}{\Sigma} \frac{\partial}{\partial r}+\frac{2}{\Sigma}\right) H=0
$$

with the general solution $H=(2 M r-\beta) / \Sigma$, where $M$ and $\beta$ are arbitrary integration constants. By applying the Boyer-Lindquist transformation $d u=d t-\left(r^{2}+a^{2}\right) d r / \Delta, d \phi=$ $d \varphi-a d r / \Delta$, where $\Delta=r^{2}+a^{2}-2 M r+\beta$, the induced metric for a rotating black hole on the brane takes the form of the Kerr-Newman solution of standard general relativity, describing a stationary and axisymmetric charged black hole. The parameter $M$ can be interpreted as the mass of the black hole, but since there is no electric charge on the brane, the parameter $\beta$, the tidal charge parameter, which can take both positive and negative values, carries the imprints of a non-local, Coulomb type interaction from the bulk. The components of the projections of the Weyl tensor from the bulk are given by $E_{t}^{t}=-E_{\varphi}^{\varphi}=$ $-\beta\left[\Sigma-2\left(r^{2}+a^{2}\right)\right] / \Sigma^{3}, E_{r}^{r}=-E_{\theta}^{\theta}=\beta / \Sigma^{2}$, and $E_{\varphi}^{t}=-2 \beta a\left(r^{2}+a^{2}\right) \sin ^{2} \theta / \Sigma^{3}$, which shows a clear analogy with the energy-momentum tensor of a charged rotating black hole in standard general relativity [19].

\section{THIN ACCRETION DISKS ONTO BLACK HOLES}

The theory of mass accretion around rotating black holes was developed for the general relativistic case by Novikov and Thorne [25]. They extended the steady-state thin disk models introduced by Shakura and Sunyaev [24] to the curved space-time, by adopting the equatorial approximation for the stationary and axisymmetric geometry. The time- and space-like Killing vector fields $(\partial / \partial t)^{\mu}$ and $(\partial / \partial \phi)^{\mu}$ describe the symmetry properties of this type of space-time, where $t$ and $r$ are the Boyer-Lyndquist time and radial coordinates, respectively.

The horizontal size of the thin disk is negligible as compared to its vertical extension, i.e, the disk height $H$, defined by the maximum half thickness of the disk, is much smaller than any characteristic radii $r$ of the disk, $H<<r$. In the steady-state accretion disk models,

the mass accretion rate $\dot{M}_{0}$ is supposed to be constant in time, and the physical quantities of the accreting matter are averaged over a characteristic time scale, e.g. $\Delta t$, and over the 
azimuthal angle $\Delta \phi=2 \pi$, for a total period of the orbits and for the height $H$. The plasma moves in Keplerian orbits around the black hole, with a rotational velocity $\Omega$, and the plasma particles have a specific energy $\widetilde{E}$, and specific angular momentum $\widetilde{L}$, which depend only on the radii of the orbits. The particles are orbiting with the four-velocity $u^{\mu}$ in a disk having an averaged surface density $\Sigma$. The accreting matter is modeled by an anisotropic fluid source, where the density $\rho_{0}$ (the specific heat is neglected), the energy flow vector $q^{\mu}$ and the stress tensor $t^{\mu \nu}$ are measured in the averaged rest-frame. The energy-momentum tensor describing this source takes the form

$$
T^{\mu \nu}=\rho_{0} u^{\mu} u^{\nu}+2 u^{(\mu} q^{\nu)}+t^{\mu \nu},
$$

where $u_{\mu} q^{\mu}=0, u_{\mu} t^{\mu \nu}=0$. The four-vectors of the energy and of the angular momentum flux are defined by

$$
-E^{\mu} \equiv T^{\mu}{ }_{\nu}(\partial / \partial t)^{\nu} \quad J^{\mu} \equiv T^{\mu}{ }_{\nu}(\partial / \partial \phi)^{\nu}
$$

respectively. The four dimensional conservation laws of the rest mass, of the energy and of the angular momentum of the plasma provide the structure equations of the thin disk. By integrating the equation of the rest mass conservation, $\nabla_{\mu}\left(\rho_{0} u^{\mu}\right)=0$, it follows that the time averaged accretion rate $\dot{M}_{0}$ is independent of the disk radius:

$$
\dot{M}_{0} \equiv-2 \pi r \Sigma u^{r}=\mathrm{const}
$$

where a dot represents the derivative with respect to the time coordinate [26]. The averaged rest mass density is defined by

$$
\Sigma(r)=\int_{-H}^{H}\left\langle\rho_{0}\right\rangle d z
$$

where $\left\langle\rho_{0}\right\rangle$ is the rest mass density averaged over $\Delta t$ and $2 \pi$. The conservation law $\nabla_{\mu} E^{\mu}=0$ of the energy can be written in an integral form as

$$
\left[\dot{M}_{0} \widetilde{E}-2 \pi r \Omega W_{\phi}^{r}\right]_{, r}=4 \pi \sqrt{-g} F \widetilde{E}
$$

where a comma denotes the derivative with respect to the radial coordinate $r$. Eq. (22) shows the balance between the energy transported by the rest mass flow, the dynamical stresses in the disk, and the energy radiated away from the surface of the disk, respectively. The torque $W_{\phi}{ }^{r}$ in Eq. (22) is given by

$$
W_{\phi}^{r}=\int_{-H}^{H}\left\langle t_{\phi}^{r}\right\rangle d z
$$


where $\left\langle t_{\phi}{ }^{r}\right\rangle$ is the $\phi-r$ component of the stress tensor, averaged over $\Delta t$ and over a $2 \pi$ angle. The law of the angular momentum conservation, $\nabla_{\mu} J^{\mu}=0$, states in its integral form the balance of the three forms of the angular momentum transport,

$$
\left[\dot{M}_{0} \widetilde{L}-2 \pi r W_{\phi}^{r}\right]_{, r}=4 \pi \sqrt{-g} F \widetilde{L}
$$

By eliminating $W_{\phi}^{r}$ from Eqs. (22) and (24), and by applying the universal energyangular momentum relation $d E=\Omega d J$ for circular geodesic orbits in the form $\widetilde{E}_{, r}=\Omega \widetilde{L}_{, r}$, the flux of the radiant energy over the disk can be expressed in terms of the specific energy, angular momentum and the angular velocity of the black hole. Then the flux integral leads to the expression of the energy flux $F(r)$, which is given by

$$
F(r)=-\frac{\dot{M}_{0}}{4 \pi \sqrt{-g}} \frac{\Omega_{, r}}{(\widetilde{E}-\Omega \widetilde{L})^{2}} \int_{r_{m s}}^{r}(\widetilde{E}-\Omega \widetilde{L}) \widetilde{L}_{, r} d r,
$$

where the no-torque inner boundary conditions were also prescribed [26]. This means that the torque vanishes at the inner edge of the disk, since the matter at the marginally stable orbit $r_{m s}$ falls freely into the black hole, and cannot exert considerable torque on the disk. The latter assumption is valid as long as strong magnetic fields do not exist in the plunging region, where matter falls into the hole.

Once the geometry of the space-time is known, we can derive the time averaged radial distribution of photon emission for accretion disks around black holes, and determine the efficiency of conversion of the rest mass into outgoing radiation. After obtaining the radial dependence of the angular velocity $\Omega$, of the specific energy $\widetilde{E}$ and of the specific angular momentum $\widetilde{L}$ of the particles moving on circular orbits around the black holes, respectively, we can compute the flux integral (25)).

Let us consider an arbitrary stationary and axially symmetric geometry,

$$
d s^{2}=g_{t t} d t^{2}+g_{t \phi} d t d \phi+g_{r r} d r^{2}+g_{\theta \theta} d \theta^{2}+g_{\phi \phi} d \phi^{2},
$$

where in the equatorial approximation $(|\theta-\pi / 2| \ll 1)$ the metric functions $g_{t t}, g_{t \phi}, g_{r r}, g_{\theta \theta}$ and $g_{\phi \phi}$ depend only on the radial coordinate $r$. The geodesic equations take the form

$$
\begin{aligned}
& \left(\frac{d t}{d \tau}\right)^{2}=\frac{\widetilde{E} g_{\phi \phi}+\widetilde{L} g_{t \phi}}{g_{t \phi}^{2}-g_{t t} g_{\phi \phi}}, \\
& \left(\frac{d \phi}{d \tau}\right)^{2}=-\frac{\widetilde{E} g_{t \phi}+\widetilde{L} g_{t t}}{g_{t \phi}^{2}-g_{t t} g_{\phi \phi}},
\end{aligned}
$$


and

$$
g_{r r}\left(\frac{d r}{d \tau}\right)^{2}=V(r)
$$

respectively, where $\tau$ is the affine parameter, and the potential term $V(r)$ is defined by

$$
V(r) \equiv-1+\frac{\widetilde{E}^{2} g_{\phi \phi}+2 \widetilde{E} \widetilde{L} g_{t \phi}+\widetilde{L}^{2} g_{t t}}{g_{t \phi}^{2}-g_{t t} g_{\phi \phi}} .
$$

For circular orbits in the equatorial plane the following conditions must hold

$$
V(r)=0, \quad V_{, r}(r)=0 .
$$

These conditions give the specific energy $\widetilde{E}$, the specific angular momentum $\widetilde{L}$ and the angular velocity $\Omega$ of particles moving on circular orbits around spinning general relativistic stars as

$$
\begin{aligned}
\widetilde{E} & =-\frac{g_{t t}+g_{t \phi} \Omega}{\sqrt{-g_{t t}-2 g_{t \phi} \Omega-g_{\phi \phi} \Omega^{2}}}, \\
\widetilde{L} & =\frac{g_{t \phi}+g_{\phi \phi} \Omega}{\sqrt{-g_{t t}-2 g_{t \phi} \Omega-g_{\phi \phi} \Omega^{2}}}, \\
\Omega & =\frac{d \phi}{d t}=\frac{-g_{t \phi, r}+\sqrt{\left(g_{t \phi, r}\right)^{2}-g_{t t, r} g_{\phi \phi, r}}}{g_{\phi \phi, r}} .
\end{aligned}
$$

The marginally stable orbit around the central object are determined by the condition

$$
V_{, r r}(r)=0
$$

Let us represent the effective potential in the form $V(r) \equiv-1+f / g$, where $f \equiv \widetilde{E}^{2} g_{\phi \phi}+$ $2 \widetilde{E} \widetilde{L} g_{t \phi}+\widetilde{L}^{2} g_{\phi \phi}$ and $g \equiv g_{t \phi}^{2}-g_{t t} g_{\phi \phi}$, respectively. Then from the condition $V(r)=0$ we obtain first $-1+f / g=0$, which implies $f=g$. From $V_{, r}(r)=0$ we obtain $\left(f_{, r} g-f g_{, r}\right) / g^{2}=$ 0 , while $V_{, r r}(r)=0$ gives $g^{-1}\left(f_{, r r}-g_{, r r}\right)=0$, since $V(r)=0$ and $V_{, r}(r)=0$. If $g \neq 0$ we have

$$
\widetilde{E}^{2} g_{\phi \phi, r r}+2 \widetilde{E} \widetilde{L} g_{t \phi, r r}+\widetilde{L}^{2} g_{t t, r r}-\left(g_{t \phi}^{2}-g_{t t} g_{\phi \phi}\right)_{, r r}=0
$$

By inserting Eqs. (32)-(33) into Eq. (36), and solving the resulting equation for $r$, we obtain the marginally stable orbits, once the metric coefficients $g_{t t}, g_{t \phi}$ and $g_{\phi \phi}$ are explicitly given.

In the case of a static and spherically symmetric geometry, given by Eq. (7), the geodesic equations for particles orbiting in the equatorial plane take the form

$$
e^{2 \nu}\left(\frac{d t}{d \tau}\right)^{2}=\widetilde{E}^{2}, e^{(\nu+\lambda)}\left(\frac{d r}{d \tau}\right)^{2}+V_{e f f}(r)=\widetilde{E}^{2}, r^{4}\left(\frac{d \phi}{d \tau}\right)^{2}=\widetilde{L}^{2},
$$


and the effective potential can be written as

$$
V(r) \equiv e^{\nu}\left(1+\frac{\widetilde{L}^{2}}{r^{2}}\right)
$$

The conditions for a stable particle orbit are again $V(r)=0$ and $V_{, r}(r)=0$. From these conditions we obtain

$$
\begin{aligned}
\Omega & =\sqrt{\frac{\nu_{, r} e^{\nu}}{2 r}}, \\
\widetilde{E} & =\frac{e^{\nu}}{\sqrt{e^{\nu}-r^{2} \Omega^{2}}}, \\
\widetilde{L} & =\frac{r^{2} \Omega}{\sqrt{e^{\nu}-r^{2} \Omega^{2}}} .
\end{aligned}
$$

At the marginally stable orbit (or the innermost stable circular orbit) $r_{m s}$ the condition $V_{, r r}(r)=0$ holds, condition from which we can derive the value of $r_{m s}$ for a specified function $\nu(r)$.

After inserting Eqs. (39)-(41) into the integral (25), and taking into account that $\sqrt{g}=$ $r^{2} e^{(\nu+\lambda) / 2}$ for $\theta=\pi / 2$, we can compute the flux $F(r)$ over the whole disk surface for each brane black hole geometry given by the metric functions $\nu(r)$ and $\lambda(r)$.

The accreting matter in the steady-state thin disk model is supposed to be in thermodynamical equilibrium. Therefore the radiation emitted by the disk surface can be considered as a perfect black body radiation, where the energy flux is given by $F(r)=\sigma T^{4}(r)$ ( $\sigma$ is the Stefan-Boltzmann constant), and the luminosity $L(\omega)$ has a black body spectrum [29]:

$$
L(\omega)=4 \pi d^{2} I(\omega)=\frac{4}{\pi} \cos \gamma \omega^{3} \int_{r_{i}}^{r_{f}} \frac{r d r}{\exp (\omega / T)-1} .
$$

Here $d$ is the distance to the source, $I(\omega)$ is the Planck distribution function, $\gamma$ is the disk inclination angle, and $r_{i}$ and $r_{f}$ indicate the position of the inner and outer edge of the disk, respectively. We take $r_{i}=r_{m s}$ and $r_{f} \rightarrow \infty$, since we expect the flux over the disk surface vanishes at $r \rightarrow \infty$ for any kind of brane black hole geometry.

The flux and the emission spectrum of the accretion disks around black holes satisfy some simple scaling relations, with respect to the simple scaling transformation of the radial coordinate, given by $r \rightarrow \bar{r}=r / M$, where $M$ is the mass of the black hole. Generally, the metric tensor coefficients are invariant with respect of this transformation, while the specific energy, the angular momentum and the angular velocity transform as $\widetilde{E} \rightarrow \widetilde{E}$, $\widetilde{L} \rightarrow M \widetilde{L}$ and $\Omega \rightarrow \bar{\Omega} / M$, respectively. The flux scales as $F(r) \rightarrow F(\bar{r}) / M^{4}$, giving the 
simple transformation law of the temperature as $T(r) \rightarrow T(\bar{r}) / M$. By also rescaling the frequency of the emitted radiation as $\omega \rightarrow \bar{\omega}=\omega / M$, the luminosity of the disk is given by $L(\omega) \rightarrow L(\bar{\omega}) / M$. On the other hand, the flux is proportional to the accretion rate $\dot{M}_{0}$, and therefore an increase in the accretion rate leads to a linear increase in the radiation emission flux from the disc.

The efficiency $\epsilon$ with which the central object converts rest mass into outgoing radiation is the other important physical parameter characterizing the properties of the accretion disks. The efficiency is defined by the ratio of two rates measured at infinity: the rate of the radiation of the energy of the photons escaping from the disk surface to infinity, and the rate at which mass-energy is transported to the black hole. If all the emitted photons can escape to infinity, the efficiency depends only on the specific energy measured at the marginally stable orbit $r_{m s}$,

$$
\epsilon=1-\widetilde{E}_{m s}
$$

For Schwarzschild black holes the efficiency is about 6\%, no matter if we consider the photon capture by the black hole, or not. Ignoring the capture of radiation by the black hole, $\epsilon$ is found to be $42 \%$ for rapidly rotating black holes, whereas the efficiency is $40 \%$ with photon capture in the Kerr potential.

\section{THIN DISK ACCRETION ONTO BRANE WORLD BLACK HOLES}

In the present Section we consider the accretion properties of several classes of brane world black holes, which have been obtained by solving the vacuum gravitational field equations for the metrics given by Eqs. (7) and (17), respectively, where the metric functions $\nu$ and $\lambda$ depend only on $r$. There are many black hole type solutions on the brane, and in the following we analyze only four particular example, including three static black hole solutions, described by various metric potentials $\nu(r)$ and $\lambda(r)$, and the rotating generalization of the Kerr black hole. All the corresponding metric functions satisfy the gravitational field equations on the brane. The energy flux $F(r)$ and the disk emission spectrum is obtained for supermassive brane world black holes, with a total mass of $2.5 \times 10^{6} M_{\odot}$ and by using a mass accretion rate of $2 \times 10^{-6} M_{\odot} \mathrm{yr}^{-1}$. The inclination angle $\gamma$ used for the calculation of the spectra is set to $\cos \gamma=1$. 


\section{A. The DMPR brane black hole}

The first brane black hole we consider is a solution of the vacuum field equations, obtained by Dadhich, Maartens, Papadopoulos and Rezania in [12], which represent the simplest generalization of the Schwarzschild solution of general relativity. We call this type of brane black hole as the DMPR black hole. For this solution the metric tensor components are given by

$$
e^{\nu}=e^{-\lambda}=1-\frac{2 M}{r}+\frac{Q}{r^{2}}
$$

where $Q$ is the so-called tidal charge parameter. In the limit $Q \rightarrow 0$ we recover the usual general relativistic case. The metric is asymptotically flat, with $\lim _{r \rightarrow \infty} \exp (\nu)=$ $\lim _{r \rightarrow \infty} \exp (\lambda)=1$. There are two horizons, given by

$$
r_{h}^{ \pm}=M \pm \sqrt{M^{2}-Q}
$$

Both horizons lie inside the Schwarzschild horizon $r_{s}=2 M, 0 \leq r_{h}^{-} \leq r_{h}^{+} \leq r_{s}$. In the brane world models there is also the possibility of a negative $Q<0$, which leads to only one horizon $r_{h+}$ lying outside the Schwarzschild horizon,

$$
r_{h+}=M+\sqrt{M^{2}+Q}>r_{s}
$$

In this case the horizon has a greater area than its general relativistic counterpart, so that bulk effects act to increase the entropy and decrease the temperature, and to strengthen the gravitational field outside the black hole.

DMPR brane black holes are characterized by the metric functions (44). If we insert $e^{\nu}$ from these equations into Eq. (38), we obtain the effective potential $V(r)$ for this type of black hole for any particle with a specific angular momentum $\widetilde{L}$, orbiting around the black hole, as a function of the total mass $M$, and of the tidal charge $Q$ of the black hole. In Fig. 1 we present the radial profile of $V(r)$ for $M / \widetilde{L}=4$, and different values of the tidal charge $Q$, running between $-M^{2}$ and $M^{2}$. For comparison we have also plotted the Schwarzschild potential, corresponding to $Q=0$.

By increasing $Q$ from zero to $M^{2}$ we also increase the potential barrier, as compared to the Schwarzschild case, whereas negative tidal charges lowers the barrier, as expected for the potential of the Reissner-Nordström type black holes. The variation of $Q$ also modifies the position of the marginally stable orbit, as shown by the shift of the cut-off in the left hand 


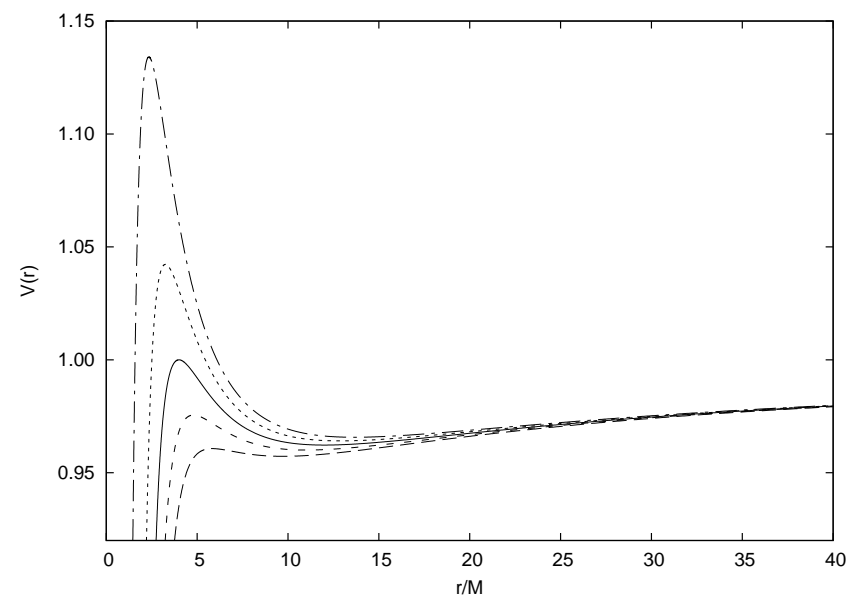

FIG. 1: The effective potential of a DMPR black hole with total mass $M$ and tidal charge $Q$ for the specific angular momentum $\widetilde{L}=4 M$. The solid line is the effective potential for a Schwarzschild black hole with the same total mass. The values of $Q$ are $Q=-M^{2}$ (long dashed line), $Q=-0.5 M^{2}$ (short dashed line), $Q=0.5 M^{2}$ (dotted line) and $Q=M^{2}$ (dot-dashed line), respectively.

side of the radial profiles of the photon flux $F(r)$ over the disk surface, which is presented in the left plot in Fig. 2.

For positive increasing tidal charges, $r_{m s}$ takes lower values, and the negative decreasing charges lead to the increase of $r_{m s}$. The position of the maximum of the radiation flux is changed similarly due to the variations of $r_{m s}$. By increasing the value of $Q$ from $-M^{2}$ to $M^{2}$, we shift the radius of the maximal flux to lower and lower values, approaching the marginally stable orbit. The vertical shift in the effective potential modifies the amplitude of the flux profile as well: higher values of the potential for $Q=0.5 M^{2}$ and $Q=M^{2}$ involve a higher specific energy of the orbiting particles, and therefore higher flux values. For $Q=-0.5 M^{2}$ and $Q=-M^{2}$, the bound state of the particles has a lower energy, and a smaller amount of energy is radiated away from the disk surface, which decreases the peak in the flux profile. In the right plot of Fig. 2 we present the variation of the flux for different values of the mass accretion rate.

These effects also cause a shift of the cut-off frequency of the black body emission spectrum of the accretion disk, as we can see in the left plot of Fig. 3. The increasing positive tidal charges shift the cut-off to higher frequencies, which produces a harder spectrum, whereas the negative decreasing values of $Q$ soften the disk spectrum by lowering the cut-off frequencies. 

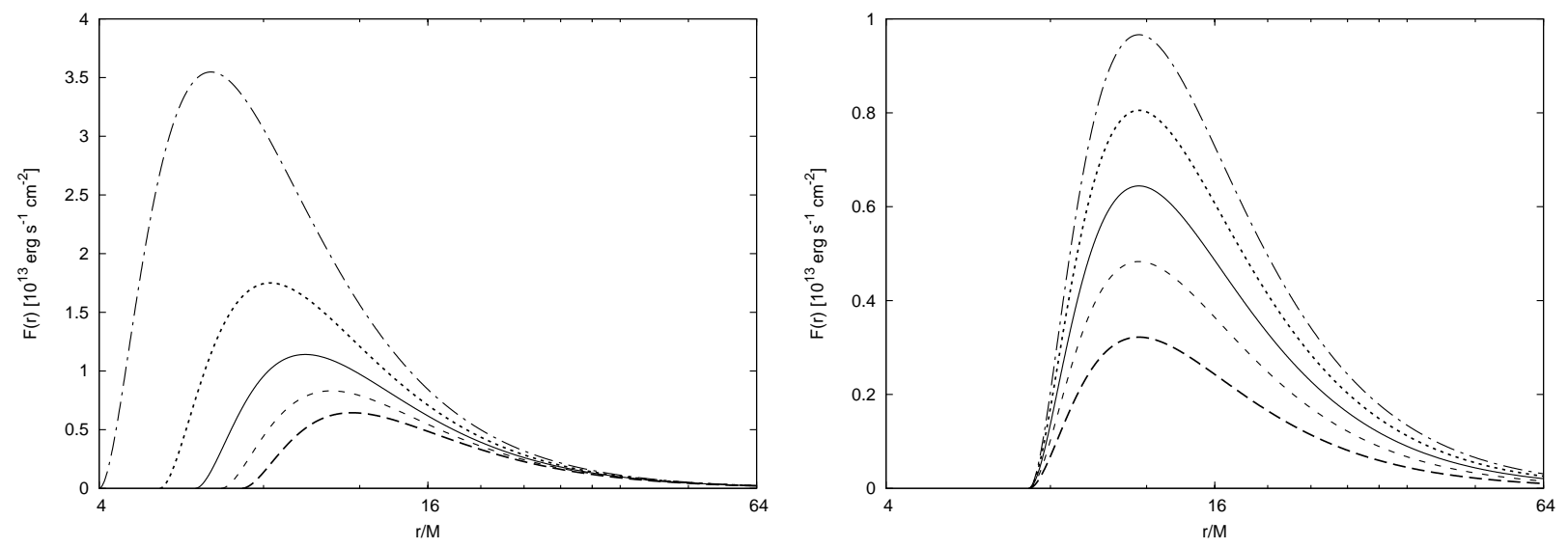

FIG. 2: Figure on the left: The time-averaged flux radiated by the accretion disk around a DMPR black hole for different values of the tidal charge parameter $Q$ : $Q=-M^{2}$ (long dashed line), $Q=-0.5 M^{2}$ (short dashed line), $Q=0.5 M^{2}$ (dotted line) and $Q=M^{2}$ (dot-dashed line), respectively. The flux for a Schwarzschild black hole is plotted with a solid line. The mass accretion rate is $2 \times 10^{-6} M_{\odot} \mathrm{yr}^{-1}$. Figure on the right: The time-averaged flux radiated by the accretion disk around a DMPR black hole and tidal charge $Q=-M^{2}$ for different values of the mass accretion rate $\dot{M}_{0}: 1.0 \times 10^{-6} M_{\odot} \mathrm{yr}^{-1}$ (long dashed line), $1.5 \times 10^{-6} M_{\odot} \mathrm{yr}^{-1}$ (short dashed line), $=2.0 \times 10^{-6} M_{\odot} \mathrm{yr}^{-1}$ (solid line), $2.5 \times 10^{-6} M_{\odot} \mathrm{yr}^{-1}$ (dotted line), and $3.0 \times 10^{-6} M_{\odot} \mathrm{yr}^{-1}$ (dot-dashed line), respectively. The total mass of the black hole is $2.5 \times 10^{6} M_{\odot}$

The variation of the emission spectrum for different values of the mass accretion rate is presented in the right plot in Fig. 3. We also present the conversion efficiency $\epsilon$ of the mass accreted by the DMPR black hole into radiation measured at infinity, which is given by Eq. (43). The value of $\epsilon$ measures the efficiency of energy generating mechanism by mass accretion. The amount of energy released by the matter leaving the marginally stable orbit, and falling into the black hole, is the binding energy $\widetilde{E}_{m s}$ of the black hole potential. For different tidal charges the values of $\widetilde{E}_{m s}$, together with the radii of the marginally stable orbits, are given in Table I, where the figures corresponding to the Schwarzschild black hole corresponds to $Q=0$. As we increase $Q$ from $-M^{2}$ to $M^{2}, r_{m s}$ is decreasing from values greater than the radius of the marginally stable orbit for the Schwarzschild geometry to lower ones. The efficiency has an opposite trend: for negative tidal charges it has smaller values than in the case of the Schwarzschild black holes, and it exceeds the latter for $Q>0$. 

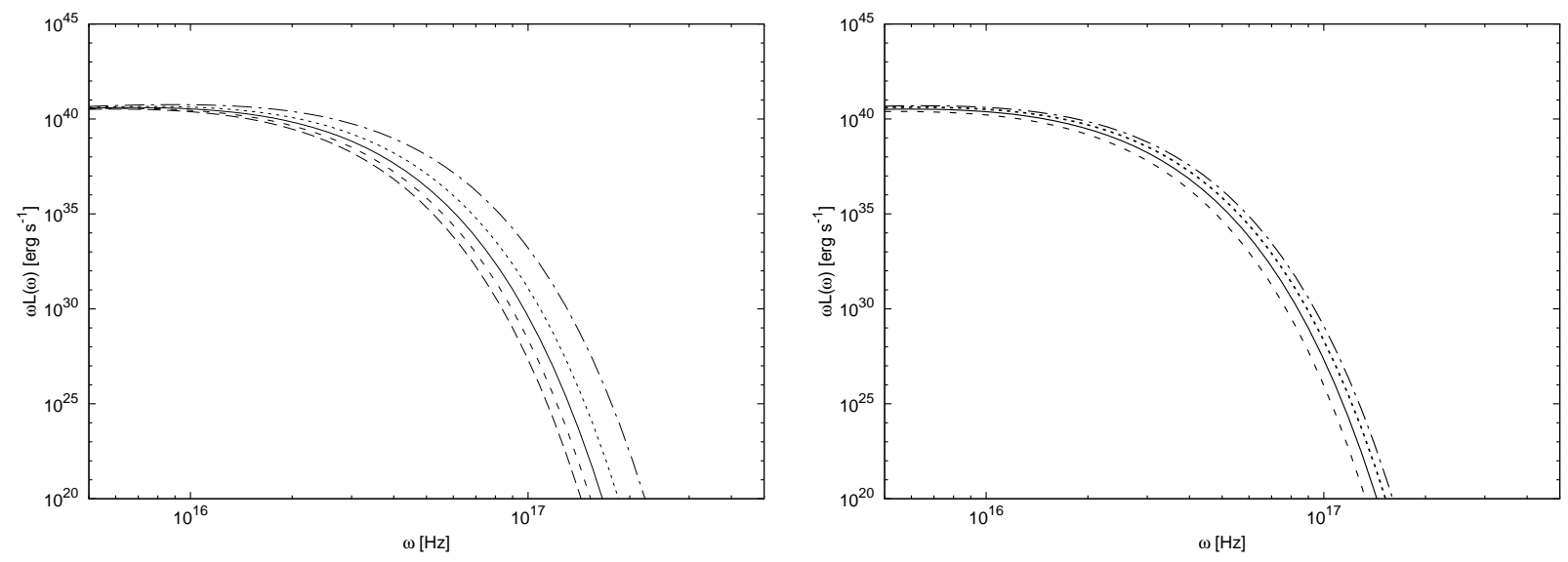

FIG. 3: Figure on the left: The emission spectrum of the accretion disk around a DMPR black hole for different values of the tidal charge parameter $Q: Q=-M^{2}$ (long dashed line), $Q=-0.5 M^{2}$ (short dashed line), $Q=0.5 M^{2}$ (dotted line) and $Q=M^{2}$ (dot-dashed line), respectively. The solid line represents the disk spectrum for a Schwarzschild black hole. The mass accretion rate is $2 \times 10^{-6} M_{\odot} \mathrm{yr}^{-1}$. Figure on the right: The emission spectrum of the accretion disk around a DMPR black hole with tidal charge $Q=-M^{2}$ for different values of the mass accretion rate $\dot{M}_{0}$ : $1.0 \times 10^{-6} M_{\odot} \mathrm{yr}^{-1}$ (long dashed line), $1.5 \times 10^{-6} M_{\odot \mathrm{yr}^{-1}}$ (short dashed line), $2.0 \times 10^{-6} M_{\odot \mathrm{yr}^{-1}}$ (solid line), $2.5 \times 10^{-6} M_{\odot \mathrm{yr}^{-1}}$ (dotted line), and $3.0 \times 10^{-6} M_{\odot} \mathrm{yr}^{-1}$ (dot-dashed line), respectively. The total mass of the black hole is $2.5 \times 10^{6} M_{\odot}$

\begin{tabular}{|c|c|c|}
\hline$Q\left[M^{2}\right]$ & $r_{m s}[M]$ & $\epsilon$ \\
\hline-1 & 7.3100 & 0.0476 \\
\hline-0.5 & 6.6949 & 0.0517 \\
\hline 0 & 6.0000 & 0.0572 \\
\hline 0.5 & 5.1695 & 0.0655 \\
\hline 1 & 4.0019 & 0.0814 \\
\hline
\end{tabular}

TABLE I: The marginally stable orbit and the efficiency for different DMPR black hole geometries. The case $Q=0$ corresponds to the standard general relativistic Schwarzschild black hole.

\section{B. The CFM brane black hole}

Two families of analytic solutions in the brane world model, parameterized by the ADM mass and the PPN parameters $\beta$ and $\gamma$, and which reduce to the Schwarzschild black hole 
for $\beta=1$, have been found by Casadio, Fabbri and Mazzacurati in [14]. We call the corresponding brane black holes as the CFM black holes.

The first class of solutions is given by

$$
e^{\nu}=1-\frac{2 M}{r}
$$

and

$$
e^{\lambda}=\frac{1-\frac{3 M}{r}}{\left(1-\frac{2 M}{r}\right)\left[1-\frac{3 M}{2 r}\left(1+\frac{4}{9} \eta\right)\right]},
$$

respectively, where $\eta=\gamma-1=2(\beta-1)$. As in the Schwarzschild case the event horizon is located at $r=r_{h}=2 M$. The solution is asymptotically flat, that is $\lim _{r \rightarrow \infty} e^{\nu} \equiv e^{\nu_{\infty}} \equiv$ $\lim _{r \rightarrow \infty} e^{\lambda} \equiv e^{\lambda_{\infty}}=1$.

The second class of solutions corresponding to brane world black holes obtained in [14] has the metric tensor components given by

$$
e^{\nu}=\left[\frac{\eta+\sqrt{1-\frac{2 M}{r}(1+\eta)}}{1+\eta}\right]^{2},
$$

and

$$
e^{\lambda}=\left[1-\frac{2 M}{r}(1+\eta)\right]^{-1}
$$

respectively. The metric is asymptotically flat. In the case $\eta>0$, the only singularity in the metric is at $r=r_{0}=2 M(1+\eta)$, where all the curvature invariants are regular. $r=r_{0}$ is a turning point for all physical curves. For $\eta<0$ the metric is singular at $r=r_{h}=2 M /(1-\eta)$ and at $r_{0}$, with $r_{h}>r_{0} . r_{h}$ defines the event horizon.

The first and second class of the CFM brane black holes are characterized by the metric potentials (47) and (49). Since for the first class of solutions the metric function $e^{\nu}$ coincides with the one of the Schwarzschild black hole, their effective potentials $V(r)$ are the same for equal total masses and fixed $\tilde{L}$. As a consequence, the specific energy, specific angular momentum and angular velocity of the particles orbiting around the first class of CFM black holes are equal to those of the particles moving at the same Keplerian orbit in the Schwarzschild potential.

However, the radiation flux from the accretion disk shows considerable differences as compared to the Schwarzschild case when the parameter $\eta$ is varied. This behavior, shown in the left plot of Fig. 4, is due to the fact that the proper volume used in the calculation of 
any integral in this spacetime depends on both the metric functions $\nu(r)$ and $\lambda(r)$, and in the flux integral given by Eq. (25) we have

$$
\sqrt{-g}=r\left[\frac{1-\frac{3 M}{r}}{1-\frac{3 M}{2 r}\left(1+\frac{4}{9} \eta\right)}\right]^{1 / 2} .
$$

Since the left hand side of Eq. (51) is decreasing for negative values of $\eta$, we obtain higher and higher flux values by decreasing this metric parameter for $\eta<0$. We have the opposite effect for $\eta>0$, where the increase of this parameter causes the proper volume to also increase, and, in turn, the amplitudes of the flux profile to decrease. Because the effective potential does not depend on $\eta$, there is no variation in the radius of the marginally stable orbit. However, a cut-off appears in the left hand side of the flux profiles for $\eta>0$, where the denominator in Eq. (51) becomes negative. This gives the criterion

$$
\frac{r}{M}<\frac{3}{2}\left(1+\frac{4 M}{9}\right)
$$

As one can see in left plot of Fig. 4, for $\eta=5$ this criterion is true for any radii greater than $r_{m s}$, but we obtain a cut-off in the left hand side of the flux profile by setting $\eta$ equal to $\eta=10$.

Although the position of the marginally stable orbit does not change for different values of $\eta$, the maximum of the radial distribution of the flux is located at higher and higher radii as we increase $\eta$. The right plot in Fig. 4 presents the variation of the flux for a fixed mass and parameter $\eta$, and for different values of the accretion rate.

The variation in the shape and the amplitude of the flux profile for different values of $\eta$ has a clear effect of shifting the cut-off frequency in the disk spectra. The left plot of Fig. 5 shows that the cut-off value shifts to higher frequencies when $\eta$ is negative, hardening the spectra. For $\eta>0$ the disk spectrum becomes softer, with lower cut-off frequencies. The effect of the variation of the mass accretion rate on the emission spectra for a fixed mass and $\eta$ is presented in the right plot of Fig. 5 .

Since the effective potential of the CFM black holes of the first class is the same as the one of the Schwarzschild geometry, their conversion efficiencies $\epsilon$ of the mass accreted by the black hole into radiation measured at infinity are also the same.

If we consider the second class of the CFM black holes, given by Eqs. (49) and (50), respectively, the variation of the parameter $\eta$ causes similar effects in the behavior of the photon flux emitted by the accretion disk and its spectrum. However, the metric function 

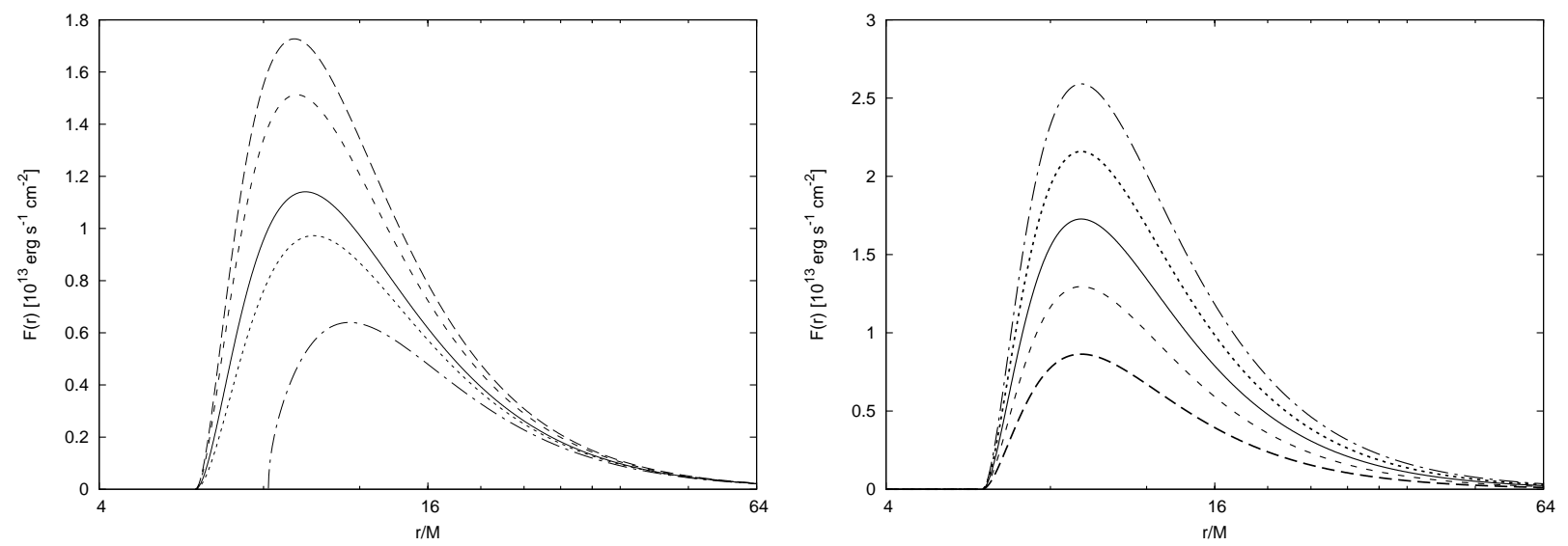

FIG. 4: Figure on the left: The time-averaged flux radiated by an accretion disk around a first class CFM black holes for various values of $\eta: \eta=-10$ (long dashed line), $\eta=-5$ (short dashed line), $\eta=5$ (dotted line) and $\eta=10$ (dot-dashed line). The flux for a Schwarzschild black hole is plotted with a solid line. The mass accretion rate is $2 \times 10^{-6} M_{\odot} \mathrm{yr}^{-1}$. Figure on the right: The time-averaged flux radiated by the accretion disk around a first class CFM black hole for $\eta=-10$ and for different values of the mass accretion rate $\dot{M}_{0}: 1.0 \times 10^{-6} M_{\odot} \mathrm{yr}^{-1}$ (long dashed line), $1.5 \times 10^{-6} M_{\odot \mathrm{yr}^{-1}}$ (short dashed line), $2.0 \times 10^{-6} M_{\odot} \mathrm{yr}^{-1}$ (solid line), $2.5 \times 10^{-6} M_{\odot} \mathrm{yr}^{-1}$ (dotted line), and $3.0 \times 10^{-6} M_{\odot} \mathrm{yr}^{-1}$ (dot-dashed line), respectively. The total mass of the black hole is $2.5 \times 10^{6} M_{\odot}$.

$\nu(r)$ now differs from the Schwarzschild black hole case, and we obtain different effective potentials for different values of the $\eta$. The radial profiles of $V(r)$ are shown in Fig. 6, where $\eta$ is set to values between -0.8 and 0.8 .

By decreasing the values of $\eta$ from zero to small negative values, we can increase the potential barrier around the black hole, and decrease the radius of the marginally stable orbit. The minimum of the effective potential is also increased. Any increment in $\eta$ from zero to small positive values causes the opposite effects for $V(r)$. Then the parameters $\eta<0$ increase the flux values and shift $r_{m s}$ to lower radii, while negative values of $\eta$ give lower fluxes with a cut-off at higher values of $r_{m s}$, as shown in the left plot of Fig. 7.

The variation of the numerical value of the radial coordinate $r$ at the position where the radiation flux takes its maximum value follows the tendency of $r_{m s}$ : with increasing $\eta$ we obtain higher and higher orbits for $F_{\max }$. These effects result in similar shifts in the disk spectrum as in the case of the CFM black holes of the first class: in the left plot of 

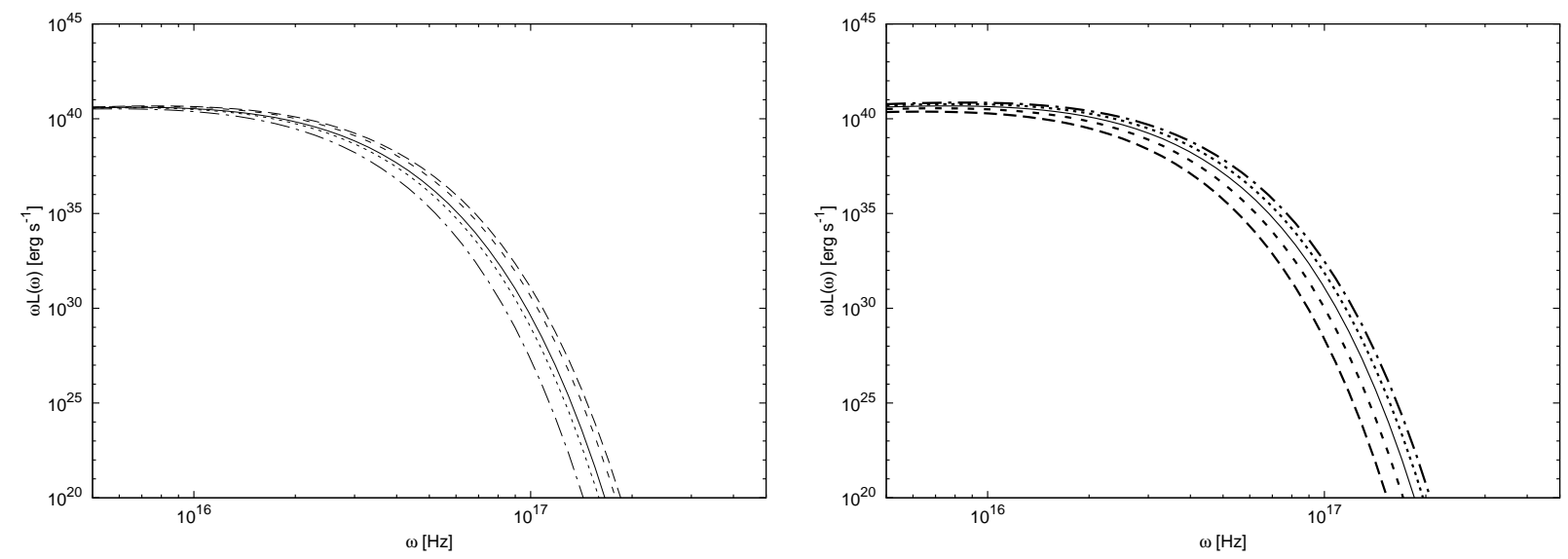

FIG. 5: Figure on the left: The emission spectrum of the accretion disk around a first class CFM black hole for different values of $\eta: \eta=-10$ (long dashed line), $\eta=-5$ (short dashed line), $\eta=5$ (dotted line) and $\eta=10$ (dot-dashed line). The spectrum for a Schwarzschild black hole is plotted with a solid line. The mass accretion rate is $2 \times 10^{-6} M_{\odot} \mathrm{yr}^{-1}$. Figure on the right: The emission spectrum of the accretion disk around a first class CFM black hole for $\eta=-10$ and for different values of the mass accretion rate $\dot{M}_{0}:=1.0 \times 10^{-6} M_{\odot} \mathrm{yr}^{-1}$ (long dashed line), $1.5 \times 10^{-6} M_{\odot} \mathrm{yr}^{-1}$ (short dashed line), $=2.0 \times 10^{-6} M_{\odot} \mathrm{yr}^{-1}$ (solid line), $2.5 \times 10^{-6} M_{\odot} \mathrm{yr}^{-1}$ (dotted line), and $3.0 \times 10^{-6} M_{\odot} \mathrm{yr}^{-1}$ (dot-dashed line), respectively. The total mass of the black hole is $2.5 \times 10^{6} M_{\odot}$.

Fig. 8 negative values of $\eta$ harden the spectrum, by shifting the frequency cut-off to higher frequencies, whereas the spectra become softer, with lower cut-off values, for increasing positive values of the metric parameter.

The right plots of Figs. 7 and 8 show the variation of the flux and emission spectra for a fixed mass and $\eta$ and for different values of the accretion rate.

We present the marginally stable orbits $r_{m s}$ and the conversion efficiency $\epsilon$ of the matter accreted by the CFM black holes of the second class into radiation measured at infinity in Table II. With the increasing values of the parameter $\eta$, the radius of the marginally stable orbit is also increasing, as already seen in the flux diagram, while $\epsilon$ drops down from values greater than 6 to lower ones. 


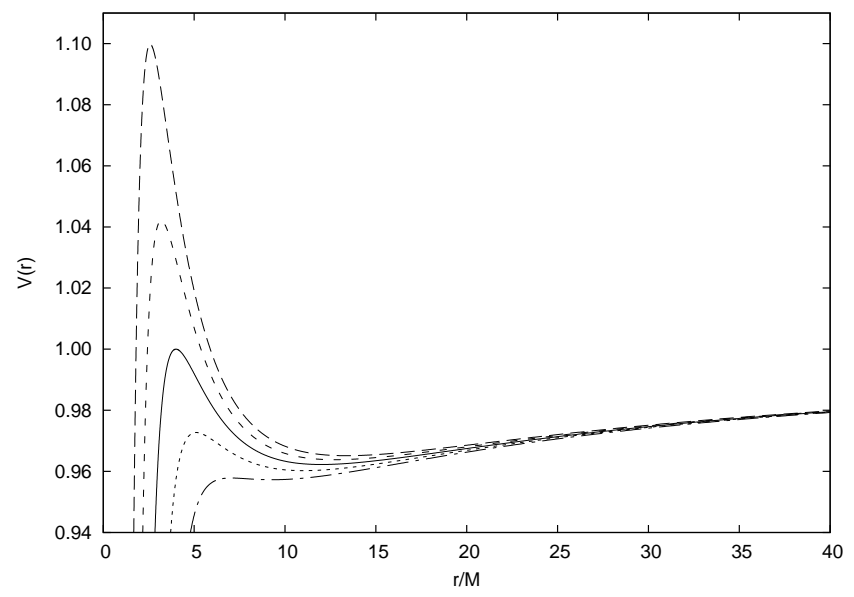

FIG. 6: The effective potential of the thin accretion disk around a CFM black hole of the second class with a total mass of $M$ and $\widetilde{L}=4 M$ for different values of the parameter $\eta$. The potential $V(r)$ corresponding to the Schwarzschild black hole is plotted with a solid line. The values of $\eta$ are $\eta=-0.8$ (long dashed line), $\eta=-0.4$ (short dashed line), $\eta=0.4$ (dotted line) and $\eta=0.8$ (dot-dashed line), respectively.

\begin{tabular}{|c|c|c|}
\hline$\eta$ & $r_{m s}[M]$ & $\epsilon$ \\
\hline-0.8 & 4.3445 & 0.0795 \\
\hline-0.4 & 5.1165 & 0.0657 \\
\hline 0 & 6.0000 & 0.0572 \\
\hline 0.4 & 6.9722 & 0.0501 \\
\hline 0.8 & 8.0091 & 0.0444 \\
\hline
\end{tabular}

TABLE II: The marginally stable orbit and the efficiency for CFM black holes of the second kind. The case $\eta=0$ corresponds to the standard general relativistic Schwarzschild black hole.

\section{The BMD brane world black hole}

Several classes of brane world black hole solutions have been obtained by Bronnikov, Melnikov and Dehnen in [18] (for short the BMD black holes). In the following we analyze the accretion properties of a particular class of these models, with metric given by

$$
e^{\nu}=\left(1-\frac{2 M}{r}\right)^{2 / s}, e^{\lambda}=\left(1-\frac{2 M}{r}\right)^{-2}
$$



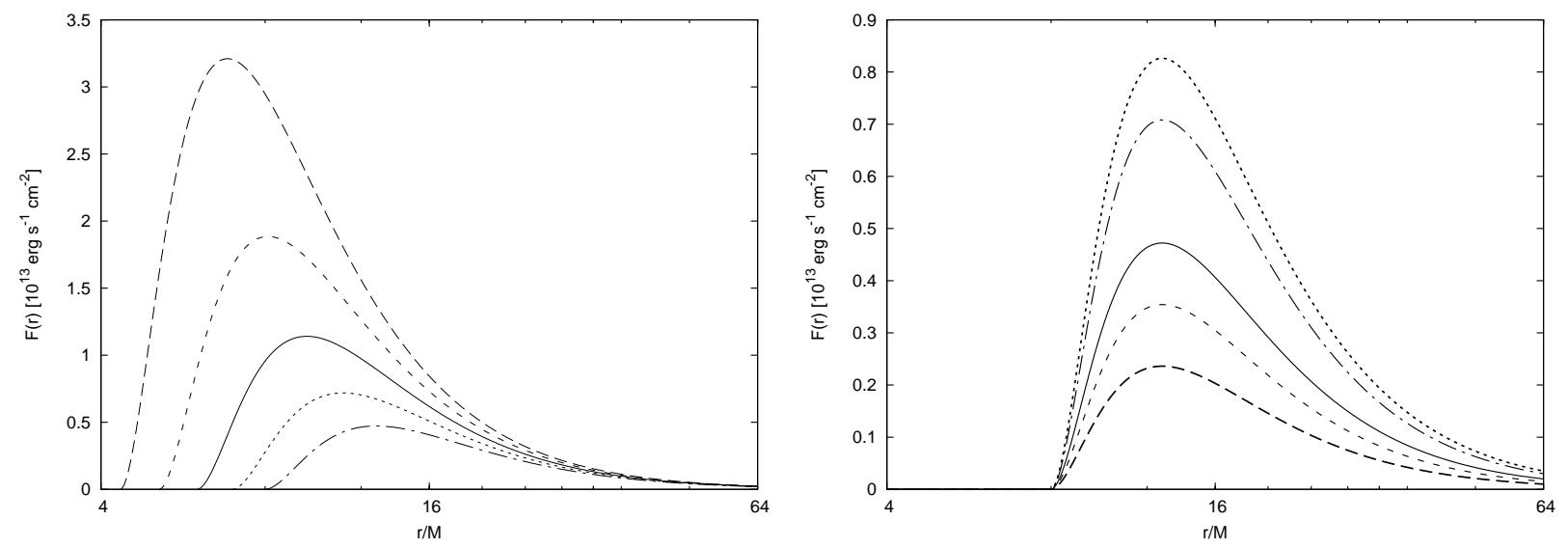

FIG. 7: Figure on the left: The time-averaged flux radiated by an accretion disk around a second class CFM black hole for different values of $\eta: \eta=-0.8$ (long dashed line), $\eta=-0.4$ (short dashed line), $\eta=0.4$ (dotted line) and $\eta=0.8$ (dot-dashed line). The flux for a Schwarzschild black hole is plotted with a solid line. The mass accretion rate is $2 \times 10^{-6} M_{\odot} \mathrm{yr}^{-1}$. Figure on the right: The time-averaged flux radiated by the accretion disk around a second class CFM black hole with the parameter $\eta=0.8$ for different values of the mass accretion rate $\dot{M}_{0}: 1.0 \times 10^{-6} M_{\odot} \mathrm{yr}^{-1}$ (long dashed line), $1.5 \times 10^{-6} M_{\odot \mathrm{yr}^{-1}}$ (short dashed line), $2.0 \times 10^{-6} M_{\odot} \mathrm{yr}^{-1}$ (solid line), $2.5 \times 10^{-6} M_{\odot} \mathrm{yr}^{-1}$ (dotted line), and $3.0 \times 10^{-6} M_{\odot} \mathrm{yr}^{-1}$ (dot-dashed line), respectively. The total mass of the black hole is $2.5 \times 10^{6} M_{\odot}$.

where $s \in \mathrm{N}$. The metric is asymptotically flat, and at $r=r_{h}=2 M$ these solutions have a double horizon.

Eqs. (53) determine the geometry of the BMD brane black holes, which have the effective potential plotted in Fig. 9. In the figure we have plotted $V(r)$ for values of $s$ between $s=5$ and $s=20$.

With increasing $s$ the potential barrier increases as well, but we obtain smaller and smaller radii for the marginally stable orbits. Although the higher values of $s$ increase the potential over the region of the stable Keplerian orbits, and the energy of the orbiting particles, as compared to the case of the Schwarzschild potential, the value of $\sqrt{-g}$ used to calculate the flux integral increases more rapidly. Therefore Eq. (25) gives smaller fluxes for higher values of $s$. This effect is shown in the left plot of Fig. 10, where we present the plots of the photon flux emitted by the accretion disk in the BMD brane black hole geometry.

The relative shift of $r_{m s}$ to lower orbits for increasing $s$ can also be well studied in the 

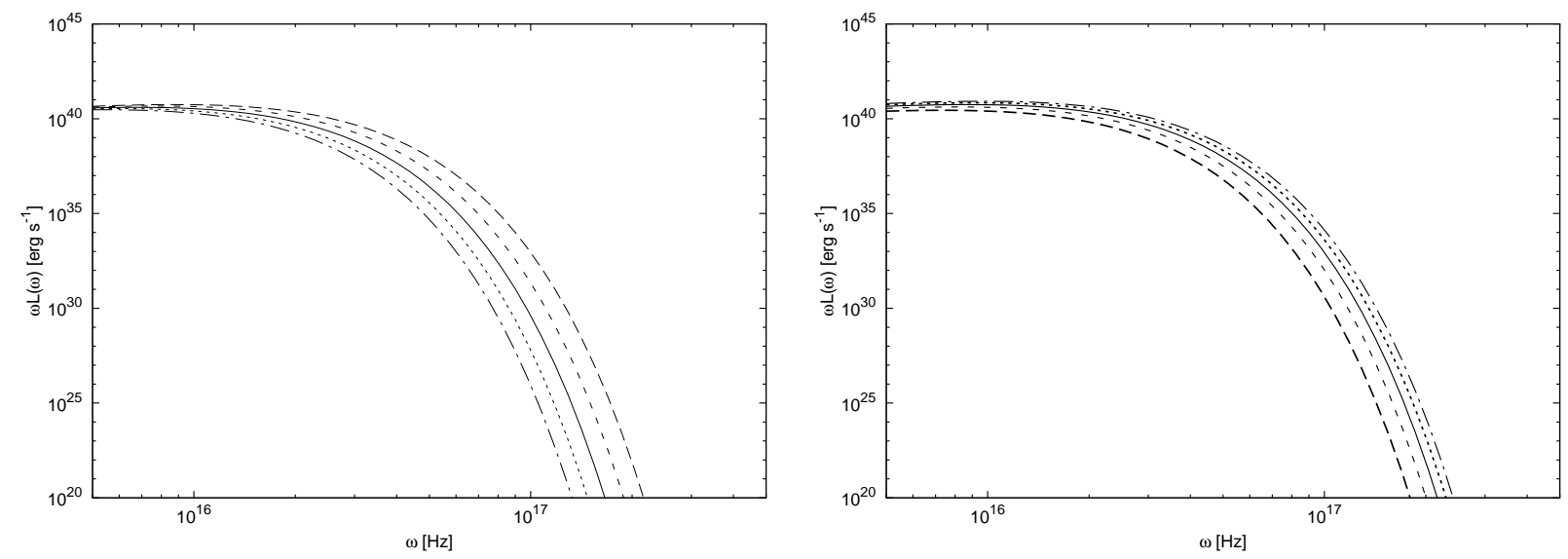

FIG. 8: Figure on the left: The emission spectrum of the thin accretion disk around a second class CFM black hole for different values of $\eta: \eta=-0.8$ (long dashed line), $\eta=-0.4$ (short dashed line), $\eta=0.4$ (dotted line), and $\eta=0.8$ (dot-dashed line). The spectrum for a Schwarzschild black hole is plotted with a solid line. The mass accretion rate is $2 \times 10^{-6} M_{\odot} \mathrm{yr}^{-1}$. Figure on the right: The emission spectrum of the accretion disk around a second class CFM black hole with parameter $\eta=10$ for different values of the mass accretion rate $\dot{M}_{0}: 1.0 \times 10^{-6} M_{\odot} \mathrm{yr}^{-1}$ (long dashed line), $1.5 \times 10^{-6} M_{\odot} \mathrm{yr}^{-1}$ (short dashed line), $2.0 \times 10^{-6} M_{\odot} \mathrm{yr}^{-1}$ (solid line), $2.5 \times 10^{-6} M_{\odot} \mathrm{yr}^{-1}$ (dotted line), and $3.0 \times 10^{-6} M_{\odot} \mathrm{yr}^{-1}$ (dot-dashed line), respectively. The total mass of the black hole is $2.5 \times 10^{6} M_{\odot}$.

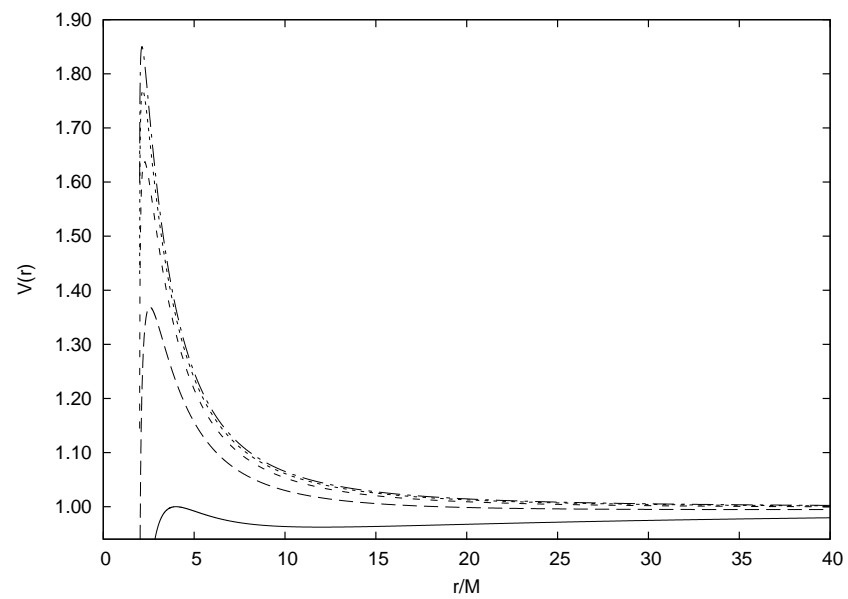

FIG. 9: The effective potential for BMD black holes of a fixed total mass $M$ for $\widetilde{L}=4 M$ and different values of $s$. The solid line is the effective potential for a Schwarzschild black hole with the same total mass $M$. The parameter $s$ is set to $s=5$ (long dashed line), $s=10$ (short dashed line), $s=15$ (dotted line) and $s=20$ (dot-dashed line). 

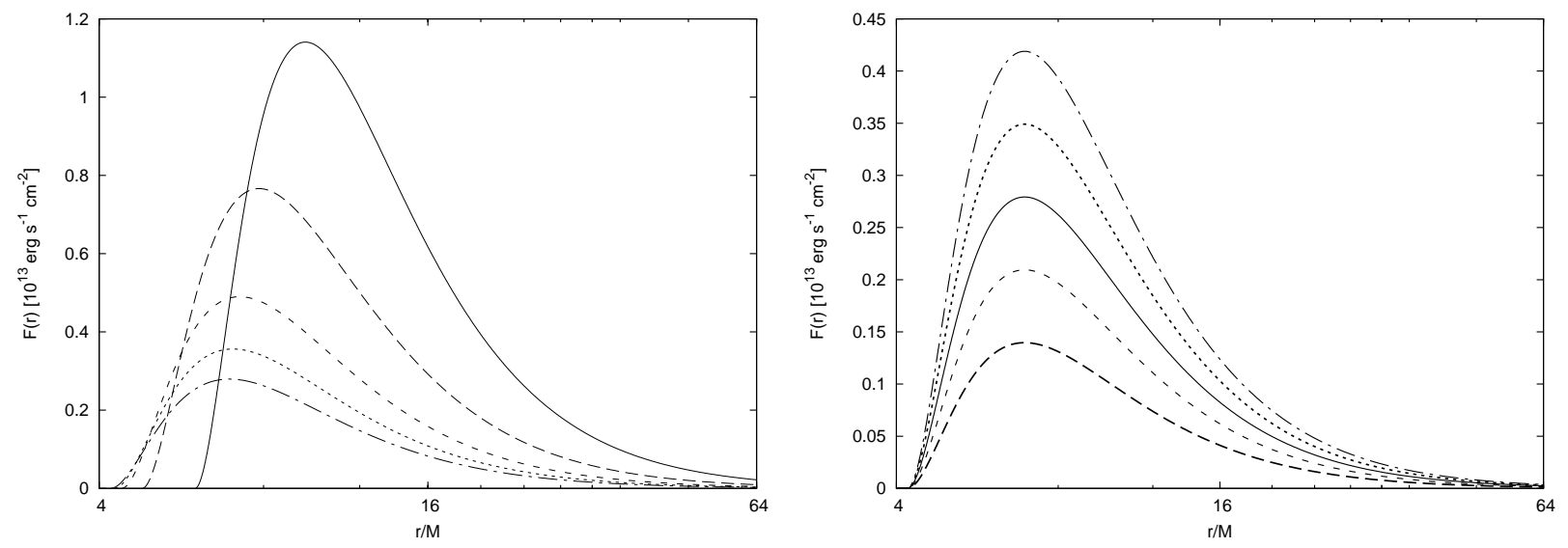

FIG. 10: Figure on the left: The time-averaged flux radiated by an accretion disk around a BMD black hole for different values of $s: s=5$ (long dashed line), $s=10$ (short dashed line), $s=15$ (dotted line) and $s=20$, respectively. The flux for a Schwarzschild black hole is plotted with a solid line. The mass accretion rate is $2 \times 10^{-6} M_{\odot} \mathrm{yr}^{-1}$. Figure on the right: The time-averaged flux radiated by the accretion disk around a BMD black hole with $s=20$ for different values of the mass accretion rate $\dot{M}_{0}: 1.0 \times 10^{-6} M_{\odot} \mathrm{yr}^{-1}$ (long dashed line), $1.5 \times 10^{-6} M_{\odot} \mathrm{yr}^{-1}$ (short dashed line), $2.0 \times 10^{-6} M_{\odot} \mathrm{yr}^{-1}$ (solid line), $2.5 \times 10^{-6} M_{\odot} \mathrm{yr}^{-1}$ (dotted line), and $3.0 \times 10^{-6} M_{\odot} \mathrm{yr}^{-1}$ (dot-dashed line), respectively. The total mass of the black hole is $2.5 \times 10^{6} M_{\odot}$.

plot. The maximum of the flux value has the same shift: by increasing $s$ the maximum of $F(r)$ is obtained at lower and lower radii. This behavior of the radiation flux results in the softening of the emission spectrum of the disk. As seen in the left plot of Fig. 11, the cut-off of the disk spectrum shifts to lower frequencies for increasing values of $s$, as compared to the ones obtained in the case of the Schwarzschild geometry. The right plots in Figs. 10 and 11 show the effect of the change of the accretion rate for a BMD brane world black hole for a fixed mass an $s$.

The marginally stable orbit and the conversion efficiency $\epsilon$ of the accreted mass into radiation measured at infinity for the BMD black holes are presented in Table III. Both $r_{m s}$ and $\epsilon$ have values less than those derived in the Schwarzschild geometry, and they exhibit a decreasing tendency as we increase the values of the parameter $s$. 

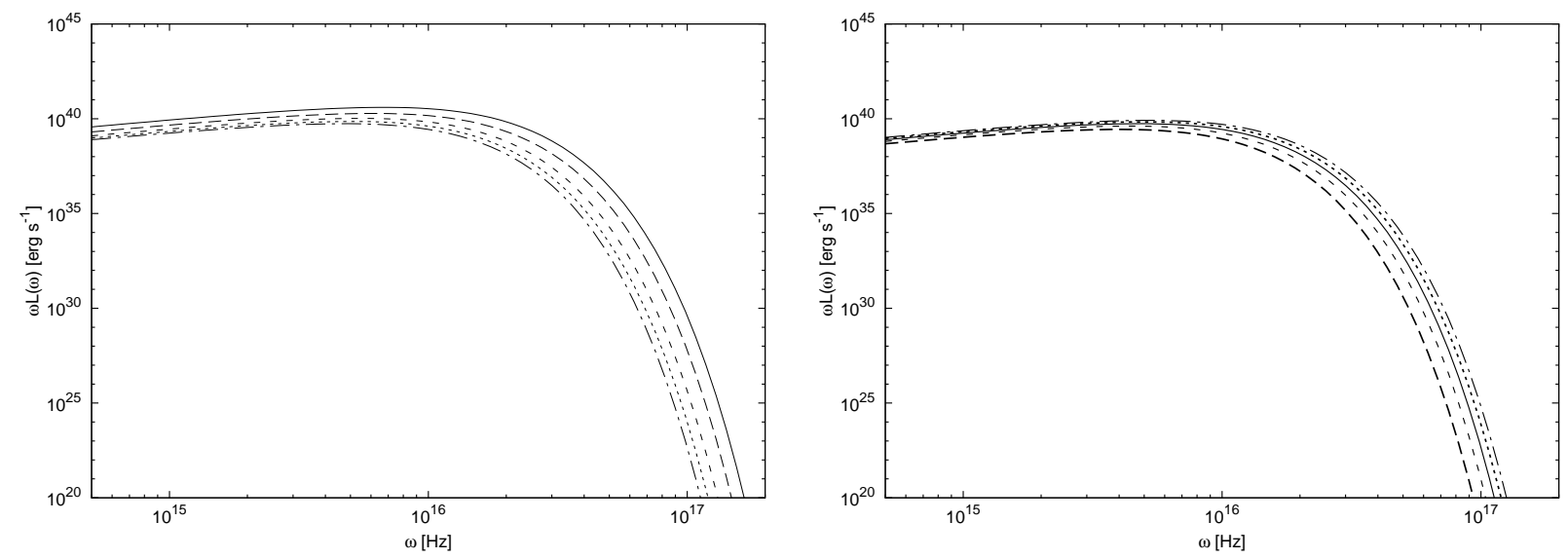

FIG. 11: Figure on the left: The emission spectrum of the thin accretion disk around a BMD black hole for different values of the parameter $s: s=5$ (long dashed line), $s=10$ (short dashed line), $s=15$ (dotted line), and $s=20$. The solid line represents the spectrum for the Schwarzschild black hole. The mass accretion rate is $2 \times 10^{-6} M_{\odot} \mathrm{yr}^{-1}$. Figure on the right: The emission spectrum of the accretion disk around a BMD black hole with parameter $s=20$ for different values of the mass accretion rate $\dot{M}_{0}: 1.0 \times 10^{-6} M_{\odot} \mathrm{yr}^{-1}$ (long dashed line), $1.5 \times 10^{-6} M_{\odot} \mathrm{yr}^{-1}$ (short dashed line), $=2.0 \times 10^{-6} M_{\odot} \mathrm{yr}^{-1}$ (solid line), $2.5 \times 10^{-6} M_{\odot} \mathrm{yr}^{-1}$ (dotted line), and $3.0 \times 10^{-6} M_{\odot} \mathrm{yr}^{-1}$ (dot-dashed line), respectively. The total mass of the black hole is $2.5 \times 10^{6} M_{\odot}$.

\begin{tabular}{|c|c|c|}
\hline$s$ & $r_{m s}[M]$ & $\epsilon$ \\
\hline 5 & 4.8005 & 0.0303 \\
\hline 10 & 4.4007 & 0.0170 \\
\hline 15 & 4.2708 & 0.0118 \\
\hline 20 & 4.2046 & 0.0090 \\
\hline
\end{tabular}

TABLE III: The marginally stable orbit and the efficiency for BMD black holes.

\section{The AG rotating brane world black hole}

The generalization of the Kerr solution of standard general relativity for rotating brane world black holes was obtained by Aliev and Gumrukcuoglu [19], and is given by

$$
d s^{2}=-\left(1-\frac{2 M r-\beta}{\Sigma}\right) d t^{2}-\frac{2 a(2 M r-\beta)}{\Sigma} \sin ^{2} \theta d t d \varphi+\frac{\Sigma}{\Delta} d r^{2}+\Sigma d \theta^{2}+
$$




$$
\left(r^{2}+a^{2}+\frac{2 M r-\beta}{\Sigma} a^{2} \sin ^{2} \theta\right) \sin ^{2} \theta d \varphi^{2} .
$$

We call this solution the AG rotating brane world black hole. The event horizon of the black hole is determined by the solution of the equation $\Delta=0$, with the largest root given by $r_{+}=M+\sqrt{M^{2}-a^{2}-\beta}$. The event horizon does exist if the condition $M^{2} \geq a^{2}+\beta$ is fulfilled. For a negative tidal charge, as $a \rightarrow M, r_{+} \rightarrow M+\sqrt{-\beta}>M$, a condition that is not allowed in standard general relativity. On the other hand for a negative tidal charge the extreme horizon $r_{+}=M$ corresponds to a black hole with rotation parameter $a$ greater than its mass $M$.

Near to the equatorial plane, $|\theta-\pi / 2| \ll 1$, by introducing the coordinate $z=r \cos \theta \approx$ $r(\theta-\pi / 2)$, the approximate form for the geometry of the AG rotating brane black hole can be written as

$$
d s^{2}=-\mathscr{D} \mathscr{A}^{-1} d t^{2}+r^{2} \mathscr{A}(d \phi-\omega d t)^{2}+\mathscr{D}^{-1} d r^{2}+d z^{2}
$$

with the metric functions

$$
\begin{aligned}
\mathscr{A} & =1+a_{*}^{2} / r_{*}^{2}+2 a_{*}^{2} / r_{*}^{3}-a_{*}^{2} \beta_{*} / r_{*}^{4}, \\
\mathscr{D} & =1-2 / r_{*}+a_{*}^{2} / r_{*}^{2}-\beta_{*} / r_{*}^{2} \\
\omega & =2 \operatorname{Mar}^{-3} \mathscr{A}^{-1},
\end{aligned}
$$

where we denoted $a_{*}=a / M, r_{*}=r / M$, and $\beta_{*}=\beta / M^{2}$, respectively. The effective potential per unit mass for the radial motion is given by

$$
V(r)=\left[\left(r^{2}+a^{2}\right) r^{2}+(2 M r-\beta) a^{2}\right] \widetilde{E}^{2}-\left(r^{2}-2 M r+\beta\right) \widetilde{L}^{2}-2 a(2 M r-\beta) \widetilde{E} \widetilde{L}-r^{2} \Delta .
$$

The variation of the potential $V(r)$ is represented, as a function of $r / M$, and for different values of the tidal charge parameter $\beta$, in Fig. 12. By increasing $\beta$ from zero to $2 M^{2}$ we also increase the potential barrier as compared with the standard general relativistic Kerr black hole case, whereas negative tidal charges lowers the potential barrier. The changes in the value of $\beta$ also modify the positions of the marginally stable orbits.

In Fig. 13 we present the flux profiles of the accretion disk in the modified Kerr geometry (55) as a function of $r / M$ and for different values of the tidal charge $\beta$ (left plot) and of the accretion rate $\dot{M}_{0}$ (right plot). The variation of the numerical value of the tidal charge determines similar modifications for the flux values as in the case of the accretion disk around 


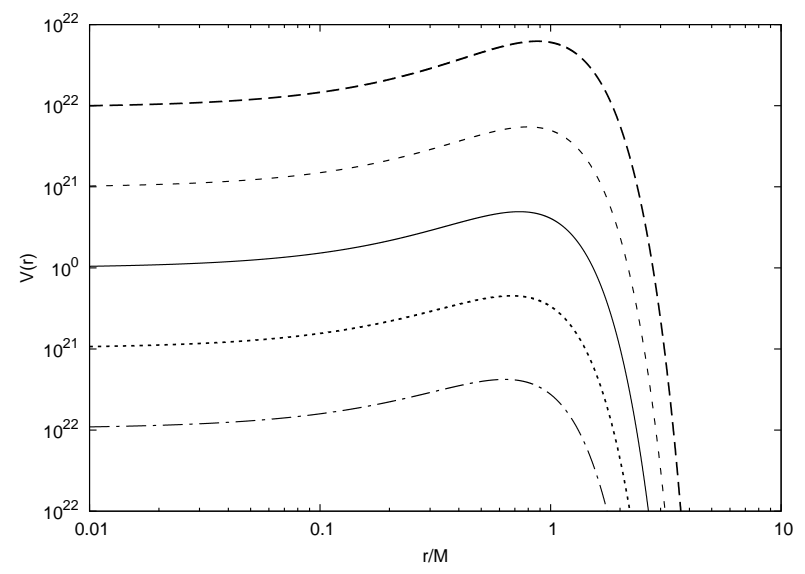

FIG. 12: The effective potential of a rotating AG brane world black hole of a total mass $2.5 \times 10^{6} M_{\odot}$ and with spin $a=0.9982$ for the specific energy $\widetilde{E}=0.8$ and the specific angular momentum $\widetilde{L}=4 M$. The solid line is the effective potential for a rotating Kerr black hole $(\beta=0)$ with the same total mass. The different values of $\beta$ are $\beta=-2 M^{2}$ (long dashed line), $\beta=-M^{2}$ (short dashed line), $\beta=M^{2}$ (dotted line) and $\beta=2 M^{2}$ (dot-dashed line), respectively.

the DMPR black holes, which can be considered as the static limit $(a=0)$ of the rotating AG black hole. The left hand plots in Figs. 13 and 2, respectively, show the same variation of the flux profiles as a function of the tidal charge. We note that since $Q$ corresponds to $-\beta$, an increase in the numerical values of $\beta$ from negative values to positive ones decreases the magnitude of the flux, and increases the radius of the marginally stable orbits. The right hand plots in Figs. 2 and 13, respectively, also exhibit the same tendency: for higher mass accretion rates the flux will be amplified as well. In Figs. 2 and 13 the cut-off values of the spectra decrease with the increasing values of $\beta$ and $-Q$, respectively. The same analogy is valid for disk spectra for the static and the rotating cases. The emission spectra in the case of the rotating AG brane world black holes are represented, for different values of the spin parameter and of the accretion rate, in Figs. 14,

By comparing Tables $\llbracket$ and $I V$ we can see the same effect of the variation of the tidal charge on the efficiency $\epsilon$ for the static DMPR and for the rotating AG brane black hole, respectively. As $\beta$ and $-Q$ increase from negative values to positive ones, the marginally stable orbits shift to higher radii, and the efficiency of the conversion of the accreting mass to radiant energy decreases, from values higher than 0.3241 (the efficiency for the standard Kerr black hole with $\left.a_{*}=0.9982\right)$ to lower ones. 

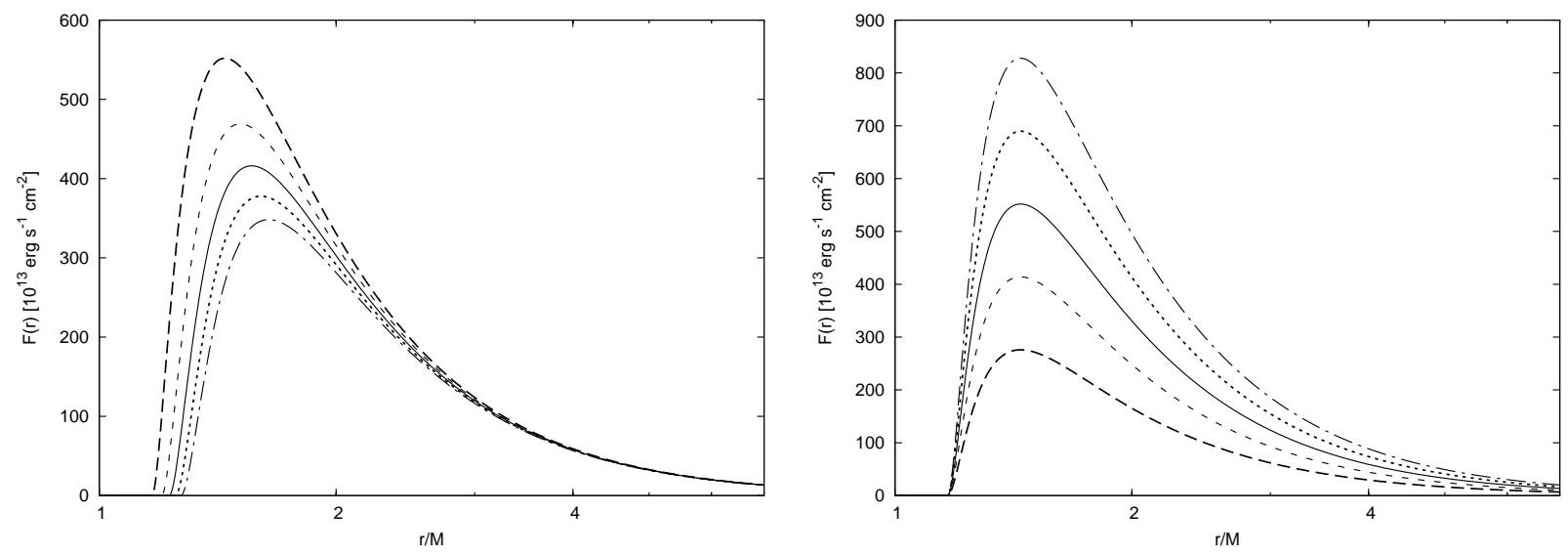

FIG. 13: Figure on the left: The time-averaged flux radiated by the accretion disk around a rotating AG brane world black hole with spin $a=0.8892$ for different values of the tidal charge parameter $\beta$ : $\beta=-2 \times 10^{-3} M^{2}$ (long dashed line), $\beta=-10^{-3} M^{2}$ (short dashed line), $\beta=10^{-3} M^{2}$ (dotted line), and $\beta=2 \times 10^{-3} M^{2}$ (dot-dashed line), respectively. The flux for a Kerr black hole with the same total mass and spin is plotted with a solid line. The mass accretion rate is $2 \times 10^{-6} M_{\odot} \mathrm{yr}^{-1}$. Figure on the right: The time-averaged flux radiated by the accretion disk around a rotating AG brane world black hole with spin $a=0.8892$ and tidal charge $\beta=-2 \times 10^{-3} M^{2}$ for different values of the mass accretion rate $\dot{M}_{0}: 1.0 \times 10^{-6} M_{\odot} \mathrm{yr}^{-1}$ (long dashed line), $1.5 \times 10^{-6} M_{\odot} \mathrm{yr}^{-1}$ (short dashed line), $=2.0 \times 10^{-6} M_{\odot} \mathrm{yr}^{-1}$ (solid line), $2.5 \times 10^{-6} M_{\odot} \mathrm{yr}^{-1}$ (dotted line), and $3.0 \times 10^{-6} M_{\odot} \mathrm{yr}^{-1}$ (dot-dashed line), respectively. The total mass of the black hole is $2.5 \times 10^{6} M_{\odot}$.

\begin{tabular}{|c|c|c|}
\hline$\beta\left[10^{-3} M^{2}\right]$ & $r_{m s}[M]$ & $\epsilon$ \\
\hline-2 & 1.1677 & 0.3449 \\
\hline-1 & 1.2019 & 0.3329 \\
\hline 0 & 1.2277 & 0.3241 \\
\hline 1 & 1.2511 & 0.3169 \\
\hline 2 & 1.2716 & 0.3109 \\
\hline
\end{tabular}

TABLE IV: The marginally stable orbit and the efficiency for different rotating AG black hole geometries for $a_{*}=0.9982$. The case $\beta=0$ corresponds to the standard general relativistic Kerr black hole. 

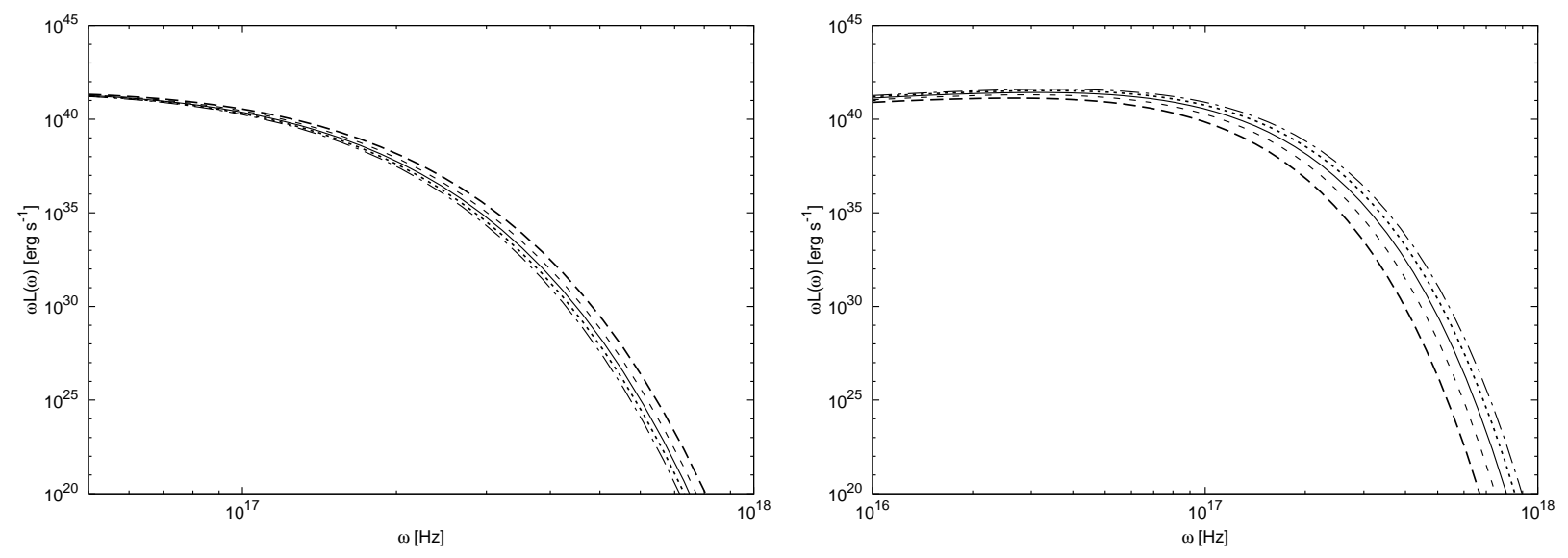

FIG. 14: Figure on the left: The emission spectrum of the accretion disk around a rotating AG brane world black hole with spin $a=0.8892$ for different values of the tidal charge parameter: $\beta=-2 \times 10^{-3} M^{2}$ (long dashed line), $\beta=-10^{-3} M^{2}$ (short dashed line), $\beta=10^{-3} M^{2}$ (dotted line), and $\beta=2 \times 10^{-3} M^{2}$ (dot-dashed line), respectively. The flux for a Kerr black hole with the same total mass and spin is plotted with a solid line. The mass accretion rate is $2 \times 10^{-6} M_{\odot} \mathrm{yr}^{-1}$. Figure on the right: The emission spectrum of the accretion disk around a rotating AG brane world black hole with spin $a=0.8892$ and tidal charge $\beta=-2 \times 10^{-3} M^{2}$ for different values of the mass accretion rate $\dot{M}_{0}: 1.0 \times 10^{-6} M_{\odot} \mathrm{yr}^{-1}$ (long dashed line), $1.5 \times 10^{-6} M_{\odot} \mathrm{yr}^{-1}$ (short dashed line), $=2.0 \times 10^{-6} M_{\odot} \mathrm{yr}^{-1}$ (solid line), $2.5 \times 10^{-6} M_{\odot} \mathrm{yr}^{-1}$ (dotted line), and $3.0 \times 10^{-6} M_{\odot} \mathrm{yr}^{-1}$ (dot-dashed line), respectively. The total mass of the black hole is $2.5 \times 10^{6} M_{\odot}$.

\section{DISCUSSIONS AND FINAL REMARKS}

In the present paper we have considered the basic physical properties of matter forming a thin accretion disk in the space-time metric of the brane world black holes. The physical parameters of the disk-effective potential, flux and emission spectrum profiles have been explicitly obtained for several classes of black holes, and for several values of the parameters characterizing the vacuum solution of the generalized field equations in the brane world models. All the astrophysical quantities related to the observable properties of the accretion disk can be obtained from the black hole metric.

There are many effective 4D solutions of the vacuum field equations on the brane, with arbitrary parameters which depend on properties of the bulk, or are simply put in by using general physical considerations. At the present moment it is theoretically not known whether 
these parameters should be universal over all brane world black holes, or whether each separate black hole may have different values of them. Conversely, there is not a single complete solution, in the sense that the metric in the bulk is uniquely known. This situation is unsatisfactory from a purely theoretical point of view, and a solution of this problem seems to be very difficult to be found. Therefore it may be useful to solve the problem of the existence and nature of the brane world black holes by investigating more closely the existing observational evidence of the black hole properties, and try to discriminate between different black hole models by using the data provided by the observational study of the astrophysical processes around black holes.

Testing strong field gravity and the detections of the possible deviations from standard general relativity, signaling the presence of new physics, remains one of the most important objectives of observational astronomy. Due to their compact nature, black holes provide an ideal environment to do this. Presently, the best constraints on the brane world black hole parameters can be obtained from the classical tests of general relativity (perihelion precession, deflection of light, and the radar echo delay, respectively). The existing observational solar system data on the perihelion shift of Mercury, on the light bending around the Sun (obtained using long-baseline radio interferometry), and ranging to Mars using the Viking lander, as applied to the DMPR black hole, can constrain the numerical values of both the bulk tidal parameter $Q$ and of the brane tension [32]. The stronger limit is obtained from the perihelion precession, $|Q| \leq 6 \times 10^{7}-5 \times 10^{8} \mathrm{~cm}^{2}$. An improvement of one order of magnitude in the observational data on Mercury's perihelion shift could provide a very precise estimate of the bulk tidal parameter, as well as of the brane tension $\lambda$.

Observations in the near-infrared (NIR) or X-ray bands have provided important information about the spin of the black holes, or the absence of a surface in stellar type black hole candidates. In the case of the source $\mathrm{Sgr} \mathrm{A}^{*}$, where the putative thermal emission due to the small accretion rate peaks in the near infrared, the results are particularly robust. However, up to now, these results have confirmed the predictions of the general relativity mainly in a qualitative way, and the observational precision achieved cannot distinguish between the different proposed theories of gravitation. However, important technological developments may allow to image black holes directly [33]. A background illuminated black hole will appear in a silhouette with radius $\sqrt{27} G M / c^{2}$, with an angular size of roughly twice that of the horizon, and may be directly observed. With an expected resolution of $20 \mu$ as, sub- 
millimeter very-long baseline interferometry (VLBI) would be able to image the silhouette cast upon the accretion flow of Sgr $A^{*}$, with an angular size of $\sim 50 \mu$ as, or M87, with an angular size of $\sim 25 \mu$ as. For a black hole embedded in an accretion flow, the silhouette will generally be asymmetric regardless of the spin of the black hole. Even in an optically thin accretion flow asymmetry will result from special relativistic effects (aberration and Doppler shifting). In principle, detailed measurements of the size and shape of the silhouette could yield information about the mass and spin of the central black hole, and provide invaluable information on the nature of the accretion flows in low luminosity galactic nuclei.

Due to the differences in the space-time structure, the brane world black holes present some important differences with respect to their disc accretion properties, as compared to the standard general relativistic Schwarzschild and Kerr cases. Therefore, the study of the accretion processes by compact objects is a powerful indicator of their physical nature. Since the conversion efficiency, as well as the flux and the spectrum of the black body radiation in the case of the brane world black holes is different as compared to the standard general relativistic case, the astrophysical determination of these physical quantities could

discriminate, at least in principle, between the different gravity theories, and give some constrains on the existence of the extra dimensions.

\section{Acknowledgments}

The work of T. H. is supported by an RGC grant of the government of the Hong Kong SAR.

[1] L. Randall and R. Sundrum, Phys. Rev. Lett., 83, 3370 (1999).

[2] L. Randall and R. Sundrum, Phys. Rev. Lett. 834690 (1999).

[3] R. Maartens, Living Reviews in Relativity 7, 1 (2004).

[4] T. Shiromizu, K. Maeda and M. Sasaki, Phys. Rev. D62, 024012 (2000).

[5] M. Sasaki, T. Shiromizu and K. Maeda, Phys. Rev. D62, 024008 (2000).

[6] R. Maartens, Phys. Rev. D62, 084023 (2000); A. Campos and C. F. Sopuerta, Phys. Rev. D63, 104012 (2001); A. Campos and C. F. Sopuerta, Phys. Rev. D64, 104011 (2001); C.-M. Chen, T. Harko and M. K. Mak, Phys. Rev. D64, 044013 (2001); D. Langlois, Phys. Rev. 
Lett. 86, 2212 (2001); C.-M. Chen, T. Harko and M. K. Mak, Phys. Rev. D64, 124017 (2001); J. D. Barrow and R. Maartens, Phys. Lett. B532, 153 (2002); C.-M. Chen, T. Harko, W. F. Kao and M. K. Mak, Nucl. Phys. B636, 159 (2002); M. Szydlowski, M. P. Dabrowski and A. Krawiec, Phys. Rev. D66, 064003 (2002); T. Harko and M. K. Mak, Class. Quantum Grav. 20, 407 (2003); C.-M. Chen, T. Harko, W. F. Kao and M. K. Mak, JCAP 0311, 005 (2003); T. Harko and M. K. Mak, Class. Quantum Grav. 21, 1489 (2004); A. N. Aliev and A. E. Gumrukcuoglu, Class. Quantum Grav. 21, 5081, (2004); M. Maziashvili, Phys. Lett. B627, 197 (2005); S. Mukohyama, Phys. Rev. D72, 061901 (2005); M. K. Mak and T. Harko, Phys. Rev. D71, 104022 (2005); L. A. Gergely, Phys. Rev. D74 024002, (2006); N. Pires, ZongHong Zhu, J. S. Alcaniz, Phys. Rev. D73, 123530 (2006); G. M. Szabo, L. A. Gergely and Z. Keresztes, PMC Physics A1, 8 (2007); Z. Keresztes, L. A. Gergely, B. Nagy and G. M. Szabo, PMC Physics A1, 4 (2007); L. A. Gergely and I. Kepiro, JCAP 0707, 007 (2007); Z. Kovacs and L. A. Gergely, Phys. Rev. D77, 024003 (2008); T. Harko and V. S. Sabau, Phys. Rev. D77, 104009 (2008); T. Harko, W. F. Choi, K. C. Wong and K. S. Cheng, JCAP 06, 002 (2008); G. De Risi, T. Harko, F. S. N. Lobo and C. S. J. Pun, arXiv:0807.3066 (2008).

[7] G. Kofinas, E. Papantonopoulos and V. Zamarias, Phys. Rev. D 66, 104028 (2002); G. Kofinas, E. Papantonopoulos and I. Pappa, Phys. Rev. D 66, 104014 (2002); D. Stojkovic, JHEP 0409, 061, 2004; D. Karasik, C. Sahabandu, P. Suranyi and L. C. R. Wijewardhana, Phys. Rev. D70, 064007 (2004); V. P. Frolov, D. V. Fursaev and D. Stojkovic, JHEP 0406, 057 (2004); R. Whisker, Phys. Rev. D71, 064004 (2005); A. S. Majumdar and N. Mukherjee, Int. J. Mod. Phys. D14, 1095 (2005); A. L. Fitzpatrick, L. Randall and T. Wiseman, JHEP 0611, 033 (2006); G. A. S. Dias, S. Gao and J. P. S. Lemos, Phys. Rev. D 75, 024030 (2007); J. Ponce de Leon, Class. Quantum Grav. 25, 075012 (2008); S. Gao and J. P. S. Lemos, Int. J. Mod. Phys. A 23, 2943 (2008).

[8] T. Harko and M. K. Mak, Phys. Rev. D69, 064020 (2004).

[9] M. K. Mak and T. Harko, Phys. Rev. D70, 024010 (2004).

[10] T. Harko and M. K. Mak, Annals of Physics 319, 471 (2005).

[11] T. Harko and K. S. Cheng, Astrophys. J. 636, 8 (2006).

[12] N. Dadhich, R. Maartens, P. Papadopoulos and V. Rezania, Phys. Lett. B487, 1 (2000).

[13] C. Germani and R. Maartens, Phys. Rev. D64, 124010 (2001).

[14] R. Casadio, A. Fabbri and L. Mazzacurati, Phys. Rev. D65, 084040 (2002). 
[15] S. Shankaranarayanan and N. Dadhich, Int. J. Mod. Phys. D13, 1095 (2004).

[16] M. Visser and D. L. Wiltshire, Phys. Rev. D67, 104004 (2003).

[17] R. Casadio and L. Mazzacurati, Mod. Phys. Lett. A18, 651 (2003).

[18] K. A. Bronnikov, V. N. Melnikov and H. Dehnen, Phys. Rev. D68, 024025 (2003).

[19] A. N. Aliev and A. E. Gumrukcuoglu, Phys. Rev. D71, 104027 (2005).

[20] A. S. Majumdar and N. Mukherjee, Int. J. Mod. Phys. D14, 1095 (2005).

[21] C. M. Urry and P. Padovani, Publ. Astron. Soc. of the Pacific 107, 803 (1995).

[22] M. Miyoshi, J. Moran, J. Herrnstein, L. Greenhill, N. Nakai, P. Diamond and M. Inoue, Nature 373, 127 (1995).

[23] A. E. Broderick and R. Narayan, Astrophys. J. 638, L21 (2006).

[24] N. I. Shakura and R. A. Sunyaev, Astron. Astrophys. 24, 33 (1973).

[25] I. D. Novikov and K. S. Thorne, in Black Holes, ed. C. DeWitt and B. DeWitt, New York: Gordon and Breach (1973).

[26] D. N. Page and K. S. Thorne, Astrophys. J. 191, 499 (1974).

[27] K. S. Thorne, Astrophys. J. 191, 507 (1974).

[28] S. Bhattacharyya, A. V. Thampan and I. Bombaci, Astron. Astrophys. 372, 925 (2001).

[29] D. Torres, Nucl. Phys. B 626, 377 (2002); F. S. Guzman, Phys. Rev. D73, 021501 (2006).

[30] Y. F. Yuan, R. Narayan and M. J. Rees, Astrophys. J. 606, 1112 (2004).

[31] C. S. J. Pun, Z. Kovacs and T. Harko, Phys. Rev. D78, 024043 (2008).

[32] C. G. Boehmer, T. Harko, F. S. N. Lobo, Class. Quantum Grav. 25, 045015 (2008)

[33] A. E. Broderick and A. Loeb, Astrophys. J. 636, L109 (2006). 